\title{
Differential Homological Algebra and General Relativity
}

\author{
Jean-Francois Pommaret \\ CERMICS, Ecole des Ponts Paris Tech, Paris, France \\ Email: jean-francois.pommaret@wanadoo.fr
}

How to cite this paper: Pommaret, J.-F. (2019) Differential Homological Algebra and General Relativity. Journal of Modern Physics, 10, 1454-1486.

https://doi.org/10.4236/jmp.2019.1012097

Received: September 12, 2019

Accepted: November 3, 2019

Published: November 6, 2019

Copyright $\odot 2019$ by author(s) and Scientific Research Publishing Inc. This work is licensed under the Creative Commons Attribution International License (CC BY 4.0).

http://creativecommons.org/licenses/by/4.0/

\begin{abstract}
In 1916, F.S. Macaulay developed specific localization techniques for dealing with "unmixed polynomial ideals" in commutative algebra, transforming them into what he called "inverse systems" of partial differential equations. In 1970, D.C. Spencer and coworkers studied the formal theory of such systems, using methods of homological algebra that were giving rise to "differential homological algebra", replacing unmixed polynomial ideals by "pure differential modules". The use of "differential extension modules" and "differential double duality" is essential for such a purpose. In particular, 0-pure differential modules are torsion-free and admit an "absolute parametrization" by means of arbitrary potential like functions. In 2012, we have been able to extend this result to arbitrary pure differential modules, introducing a "relative parametrization" where the potentials should satisfy compatible "differential constraints". We recently noticed that General Relativity is just a way to parametrize the Cauchy stress equations by means of the formal adjoint of the Ricci operator in order to obtain a "minimum parametrization" by adding sufficiently many compatible differential constraints, exactly like the Lorenz condition in electromagnetism. In order to make this difficult paper rather self-contained, these unusual purely mathematical results are illustrated by many explicit examples, two of them dealing with contact transformations, and even strengthening the comments we recently provided on the mathematical foundations of General Relativity and Gauge Theory. They also bring additional doubts on the origin and existence of gravitational waves.
\end{abstract}

\section{Keywords}

Homological Algebra, Extension Module, Torsion-Free Module, Pure Differential Module, Purity Filtration, Involution, Electromagnetism, General Relativity 


\section{Introduction}

The main purpose of this paper is to prove how apparently totally abstract mathematical tools, ranging among the most difficult ones existing in differential geometry and homological algebra today, can also become useful and enlighten many engineering or physical concepts (see the review Zbl 1079.93001 for the only application to control theory).

In the second section, we first sketch and then recall, with more specific references, the main (difficult) mathematical results on differential extension modules and differential double duality that are absolutely needed in order to understand the purity concept and, in particular, the so-called purity filtration of a differential module ([1] [2] [3] [4]). We also explain the unexpected link existing between involutivity and purity allowing to exhibit a relative parametrization of a pure differential module, even defined by a system of linear PD equations with coefficients in a non-constant differential field $K$. It is important to notice that the reduced Spencer form, which is used for such a purpose, generalizes the Kalman form existing for an OD classical control system and we shall illustrate this fact.

The third section will present for the first time a few explicit motivating academic examples in order to illustrate the above mathematical results, in particular the unexpected striking situations met in the study of contact and unimodular contact structures.

In the fourth section, we finally provide examples of applications, studying the mathematical foundations of OD/PD control theory (CT) ([3] [5]), electromagnetism (EM) ([6] [7]) and general relativity (GR) ([8] [9] [10]). Most of these examples can be now used as test examples for certain computer algebra packages recently developed for such a purpose ([11] [12]).

\section{Mathematical Tools}

Let $D=K\left[d_{1}, \cdots, d_{n}\right]=K[d]$ be the ring of differential operators with coefficients in a differential field $K$ of characteristic zero, that is such that $\mathbb{Q} \subset K$, with $n$ commuting derivations $\partial_{1}, \cdots, \partial_{n}$ and commutation relations

$d_{i} a=a d_{i}+\partial_{i} a, \forall a \in K$. If $y^{1}, \cdots, y^{m}$ are $m$ differential indeterminates, we may identify $D y^{1}+\cdots+D y^{m}=D y$ with $D^{m}$ and consider the finitely presented left differential module $M={ }_{D} M$ with presentation $D^{p} \rightarrow D^{m} \rightarrow M \rightarrow 0$ determined by a given linear multidimensional system with $n$ independent variables, $m$ unknowns and $p$ equations. Applying the functor $\operatorname{hom}_{D}(\bullet, D)$, we get the exact sequence $0 \rightarrow$ hom $_{D}(M, D) \rightarrow D^{m} \rightarrow D^{p} \rightarrow N_{D} \rightarrow 0$ of right differential modules that can be transformed by a side-changing functor to an exact sequence of finitely generated left differential modules. This new presentation corresponds to the formal adjoint $a d(\mathcal{D})$ of the linear differential operator $\mathcal{D}$ determined by the initial presentation but now with $p$ unknowns and $m$ equations, obtaining therefore a new finitely generated left differential module $N={ }_{D} N$ and we may consider $\operatorname{hom}_{D}(M, D)$ as the module of equations of the compatibility 
conditions $(\mathrm{CC})$ of $a d(\mathcal{D})$, a result which is not evident at first sight (see [3] [13]). Using now a maximum free submodule $0 \rightarrow D^{l} \rightarrow \operatorname{hom}_{D}(M, D)$ or, equivalently, a maximum number of differentially linearly independent $C C$, and repeating this standard procedure while using the well known facts that the cokernel of this monomorphism is a torsion module and $\operatorname{ad}(\operatorname{ad}(\mathcal{D}))=\mathcal{D}$, we obtain therefore an embedding $0 \rightarrow \operatorname{hom}_{D}\left(\operatorname{hom}_{D}(M, D), D\right) \rightarrow D^{l}$ of left differential modules for a certain integer $1 \leq l<m$ because $K$ is a field and thus $D$ is a Noetherian bimodule over itself, a result leading to $l=r k_{D}\left(h_{D}(M, D)\right)=r k_{D}(M)<m$ as in ([14], p. 341, [15] p. 1228) (see Section 3 for the definition of the differential rank $\left.r k_{D}\right)$. Now, the kernel of the map $\epsilon: M \rightarrow \operatorname{hom}_{D}\left(\operatorname{hom}_{D}(M, D), D\right): m \rightarrow \epsilon(m)(f)=f(m), \forall f \in \operatorname{hom}_{D}(M, D)$ is the torsion submodule $t(M)=\{m \in M \mid \exists 0 \neq P \in D, P m=0\}$ and $\epsilon$ is injective if and only if $M$ is torsion-free, that is $t(M)=0$. In that case, we obtain by composition an embedding $0 \rightarrow M \rightarrow D^{l}$ of $M$ into a free module (that can also be obtained by localization if we introduce the ring of fractions $S^{-1} D=D S^{-1}$ when $S=D-\{0\}$ ). This result is quite important for applications as it provides a (minimal) parametrization of the linear differential operator $D$ and amounts to the controllability of a classical control system when $n=1$ ([3] [16]). This parametrization will be called an "absolute parametrization" as it only involves arbitrary "potential-like" functions (see [4] [8] [9] [15] [17] [18] [19] [20] for more details and examples, in particular the fact that the Einstein equations cannot be parametrized).

It is however essential to notice that such an approach is leading to a "vicious circle" because the only constructive way to check whether $M$ is torsion-free or not is to use the differential double duality. For this reason, we briefly recall the five steps of the corresponding test allowing to know whether a given differential system or operator can be parametrized or not:

- STEP 1: Write down the system in the form of a differential operator $\mathcal{D}_{1}$.

- STEP 2: Construct its formal adjoint $a d\left(\mathcal{D}_{1}\right)$.

- STEP 3: Construct the generating CC of such an operator as a new operator and use the fact that $\operatorname{ad}(\operatorname{ad}(P))=P, \forall P \in D$ in order to denote it by $\operatorname{ad}(\mathcal{D})$.

- STEP 4: Write down $\mathcal{D}=\operatorname{ad}(\operatorname{ad}(\mathcal{D}))$.

- STEP 5: As $\operatorname{ad}(\mathcal{D}) \circ a d\left(\mathcal{D}_{1}\right)=a d\left(\mathcal{D}_{1} \circ \mathcal{D}\right)=a d(0)=0 \Leftrightarrow \mathcal{D}_{1} \circ \mathcal{D}=0$, we just need to check whether $\mathcal{D}_{1}$ generates the CC of $\mathcal{D}$ or not.

If "yes", we shall say that $\mathcal{D}_{1}$ is parametrized by $\mathcal{D}$.

If " $n o$ ", we shall say that $\mathcal{D}_{1}$ cannot be parametrized.

The purpose of this paper is to extend such a result to a much more general situation, that is when $M$ is not torsion-free, by using unexpected results first found by F.S. Macaulay in 1916 ([21]) through his study of "inverse systems" for "unmixed polynomial ideals".

Introducing $t_{r}(M)=\{m \in M \mid c d(D m)>r\}$ where the codimension of $D m$ is $n$ minus the dimension of the characteristic variety determined by $m$ in the cor- 
responding system for one unknown, we may define the purity filtration as in ([1] [3] [4]):

$$
0=t_{n}(M) \subseteq t_{n-1}(M) \subseteq \cdots \subseteq t_{1}(M) \subseteq t_{0}(M)=t(M) \subseteq M
$$

The module $M$ is said to be $r$-pure if $t_{r}(M)=0, t_{r-1}(M)=M$ or, equivalently, if $c d(M)=c d(N)=r, \forall N \subset M$ and a torsion-free module is a 0 -pure module. Moreover, when $K=k=c s t(K)$ is a field of constants and $m=1$, a pure module is unmixed in the sense of Macaulay, that is defined by an ideal having an equidimensional primary decomposition.

Example 2.1: As an elementary example with $K=k=\mathbb{Q}, m=1, n=2$, $p=2$, the differential module defined by $d_{22} y=0, d_{12} y=0$ is not pure because $z^{\prime}=d_{2} y$ satisfies $d_{2} z^{\prime}=0, \quad d_{1} z^{\prime}=0$ while $z^{\prime \prime}=d_{1} y$ only satisfies $d_{2} z^{\prime \prime}=0$ and $\left(\left(\chi_{2}\right)^{2}, \chi_{1} \chi_{2}\right)=\left(\chi_{2}\right) \cap\left(\chi_{1}, \chi_{2}\right)^{2}$. We obtain therefore the purity filtration $0=t_{2}(M) \subset t_{1}(M) \subset t_{0}(M)=t(M)=M$ with strict inclusions as $0 \neq z^{\prime} \in t_{1}(M)$ while $z^{\prime \prime} \in t_{0}(M)$ but $z^{\prime \prime} \notin t_{1}(M)$.

From the few (difficult) references ([1] [2] [3] [4] [5] [22]-[31]) dealing with the extension modules $\operatorname{ext}^{r}(M)=\operatorname{ext}_{D}^{r}(M, D)$ and purity in the framework of differential homological algebra, it is known that $M$ is $r$-pure if and only if there is an embedding $0 \rightarrow M \rightarrow \operatorname{ext}^{r}\left(\operatorname{ext}^{r}(M)\right)$. Indeed, the case $r=0$ is exactly the one already considered because $\operatorname{ext}^{0}(M)=\operatorname{ext}_{D}^{0}(M, D)=\operatorname{hom}_{D}(M, D)$ and the ker/coker exact sequence ([4] [5]):

$$
0 \rightarrow \operatorname{ext}^{1}(N) \rightarrow M \rightarrow \operatorname{ext}^{0}\left(\operatorname{ext}^{0}(M)\right) \rightarrow \operatorname{ext}^{2}(N) \rightarrow 0
$$

allows to test the torsion-free property of $M$ in actual practice by using the double-duality formula $t(M)=\operatorname{ext}^{1}(N)$ as in ([3] [5]).

Independently of the previous results, the following procedure, where one may have to change linearly the independent variables if necessary, is the heart towards the next effective definition of involution. It is intrinsic even though it must be checked in a particular coordinate system called $\delta$-regular ([32] [33] [34]) and is quite simple for first order systems without zero order equations.

- Equations of class $n$ : Solve the maximum number $\beta_{q}^{n}$ of equations with respect to the jets of order $q$ and class $n$. Then call $\left(x^{1}, \cdots, x^{n}\right)$ multiplicative variables.

- Equations of class $i \geq 1$ : Solve the maximum number $\beta_{q}^{i}$ of remaining equations with respect to the jets of order $q$ and class $i$. Then call $\left(x^{1}, \cdots, x^{i}\right)$ multiplicative variables and $\left(x^{i+1}, \cdots, x^{n}\right)$ non-multiplicative variables.

- Remaining equations of order $\leq q-1$ : Call $\left(x^{1}, \cdots, x^{n}\right)$ non-multiplicative variables.

In actual practice, we shall use a Janet tabular where the multiplicative "variables" are represented by their index in upper left position while the non-multiplicative variables are represented by dots in lower right position ([3] [32] [35]) (compare to ([36]).

DEFINITION 2.2: A system of PD equations is said to be involutive if its first prolongation can be achieved by prolonging its equations only with respect to 
the corresponding multiplicative variables. In that case, we may introduce the Cartan characters $\alpha_{q}^{i}=m \frac{(q+n-i-1) !}{(q-1) !(n-i) !}-\beta_{q}^{i}$ for $i=1, \cdots, n$ and we have $\operatorname{dim}\left(g_{q}\right)=\sum \alpha_{q}=\alpha_{q}^{1}+\cdots+\alpha_{q}^{n}$ and $\operatorname{dim}\left(g_{q+1}\right)=\sum i \alpha_{q}^{i}=1 \alpha_{q}^{1}+\cdots+n \alpha_{q}^{n}$. Moreover, one can exhibit the Hilbert polynomial $\operatorname{dim}\left(R_{q+r}\right)$ in $r$ with leading term $(\alpha / d !) r^{d}$ with $d \leq n$ when $\alpha$ is the smallest non-zero character in the case of an involutive symbol. Such a prolongation allows to compute in a unique way the principal ( $p r i)$ jets from the parametric ( par) other ones. This definition may also be applied to nonlinear systems as well.

REMARK 2.3: For an involutive system with $\beta=\beta_{q}^{n}<m$, then $\left(y^{\beta+1}, \cdots, y^{m}\right)$ can be given arbitrarily and may constitute the input variables in control theory, though it is not necessary to make such a choice. In this case, the intrinsic number $\alpha=\alpha_{q}^{n}=m-\beta>0$ is called the $n$-character and is the system counterpart of the so-called "differential transcendence degree" in differential algebra and the "rank" in module theory. As we shall see in the next Section, the smallest non-zero character and the number of zero characters are intrinsic numbers that can most easily be known by bringing the system to involution and we have $\alpha_{q}^{1} \geq \cdots \geq \alpha_{q}^{n} \geq 0$.

In the situation of the last remark, the following procedure will generalize for PD control systems the well known first order Kalman form of OD control systems where the derivatives of the input do not appear ([3], VI, Remark 1.14, $\mathrm{p}$ 802). For this, we just need to modify the Spencer form and we provide the procedure that must be followed in the case of a first order involutive system with no zero order equation, for example an involutive Spencer form.

- Look at the equations of class $n$ solved with respect to $y_{n}^{1}, \cdots, y_{n}^{\beta}$.

- Use integrations by parts like:

$$
y_{n}^{1}-a(x) y_{n}^{\beta+1}=d_{n}\left(y^{1}-a(x) y^{\beta+1}\right)+\partial_{n} a(x) y^{\beta+1}=\bar{y}_{n}^{1}+\partial_{n} a(x) y^{\beta+1}
$$

- Modify $y^{1}, \cdots, y^{\beta}$ to $\bar{y}^{1}, \cdots, \bar{y}^{\beta}$ in order to "absorb" the various $y_{n}^{\beta+1}, \cdots, y_{n}^{m}$ only appearing in the equations of class $n$.

We have the following unexpected result providing what we shall call a reduced Spencer form:

THEOREM 2.4: The new equations of class $n$ contain $y^{1}, \cdots, y^{\beta}$ and their jets but only contain $y_{i}^{\beta+1}, \cdots, y_{i}^{m}$ with $0 \leq i \leq n-1$ while the equations of class $1, \cdots, n-1$ no longer contain $y^{\beta+1}, \cdots, y^{m}$ and their jets. Accordingly, as we shall see in the next section, any torsion element, if it exists, only depends on $\bar{y}^{1}, \cdots, \bar{y}^{\beta}$.

If $\chi_{1}, \cdots, \chi_{n}$ are $n$ algebraic indeterminates or, in a more intrinsic way, if $\chi=\chi_{i} d x^{i} \in T^{*}$ is a covector and $\mathcal{D}: E \rightarrow F: \xi \rightarrow a_{k}^{\tau \mu}(x) \partial_{\mu} \xi^{k}(x)$ is a linear involutive operator of order $q$, we may introduce the characteristic matrix $a(x, \chi)=\left(a_{k}^{\tau \mu}(x) \chi_{\mu},|\mu|=\mu_{1}+\cdots+\mu_{n}=q\right)$ and the resulting map $\sigma_{\chi}(\mathcal{D}): E \rightarrow F$ is called the symbol of $\mathcal{D}$ at $\chi$. Then there are two possibilities:

- If $\max _{\chi} r k\left(\sigma_{\chi}(\mathcal{D})\right)<m \Leftrightarrow \alpha_{q}^{n}>0$ : the characteristic matrix fails to be injec- 
tive for any covector.

- If $\max _{\chi} r k\left(\sigma_{\chi}(\mathcal{D})\right)=m \Leftrightarrow \alpha_{q}^{n}=0$ : the characteristic matrix fails to be injective if and only if all the determinants of the $m \times m$ submatrices vanish. However, one can prove that this algebraic ideal $\mathfrak{a} \in K[\chi]$ is not intrinsically defined and must be replaced by its radical $\operatorname{rad}(\mathfrak{a})$ made by all polynomials having a power in $\mathfrak{a}$. This radical ideal is called the characteristic ideal of the operator.

DEFINITION 2.5: For each $x \in X$, the algebraic set defined by the characteristic ideal is called the characteristic set of $\mathcal{D}$ at $x$ and $V=\bigcup_{x \in X} V_{x}$ is called the characteristic set of $\mathcal{D}$ while we keep the word "variety" for an irreducible algebraic set defined by a prime ideal.

One has the following important theorem ([3] [13]) that will play an important part later on:

THEOREM 2.6: (Hilbert-Serre) The dimension $d(V)$ of the characteristic set, that is the maximum dimension of the irreducible components, is equal to the number of non-zero characters while the codimension $c d(V)=n-d(V)$ is equal to the number of zero characters, that is to the number of " $f u l l$ " classes in the Janet tabular of an involutive system.

If $P=a^{\mu} d_{\mu} \in D=K[d]$ with implicit summation on the multi-index, the highest value of $|\mu|$ with $a^{\mu} \neq 0$ is called the order of the operator $P$ and the ring $D$ with multiplication $(P, Q) \rightarrow P \circ Q=P Q$ is filtred by the order $q$ of the operators. We have the filtration $0 \subset K=D_{0} \subset D_{1} \subset \cdots \subset D_{q} \subset \cdots \subset D_{\infty}=D$. Moreover, it is clear that $D$, as an algebra, is generated by $K=D_{0}$ and $T=D_{1} / D_{0}$ with $D_{1}=K \oplus T$ if we identify an element $\xi=\xi^{i} d_{i} \in T$ with the vector field $\xi=\xi^{i}(x) \partial_{i}$ of differential geometry, but with $\xi^{i} \in K$ now. It follows that $D={ }_{D} D_{D}$ is a bimodule over itself, being at the same time a left $D$-module by the composition $P \rightarrow Q P$ and a right $D$-module by the composition $P \rightarrow P Q$. We define the adjoint map $a d: D \rightarrow D^{o p}: P=a^{\mu} d_{\mu} \rightarrow a d(P)=(-1)^{|\mu|} d_{\mu} a^{\mu}$ and we have $\operatorname{ad}(\operatorname{ad}(P))=P$. It is easy to check that $\operatorname{ad}(P Q)=a d(Q) a d(P), \forall P, Q \in D$. Such a definition can also be extended to any matrix of operators by using the transposed matrix of adjoint operators (see [3] [5] [8] [17] [20] [37] [38] for more details and applications to control theory and mathematical physics).

Accordingly, if $y=\left(y^{1}, \cdots, y^{m}\right)$ are differential indeterminates, then $D$ acts on $y^{k}$ by setting $d_{\mu} y^{k}=y_{\mu}^{k}$ with $d_{i} y_{\mu}^{k}=y_{\mu+1_{i}}^{k}$ and $y_{0}^{k}=y^{k}$. We may therefore use the jet coordinates in a formal way as in the previous section. Therefore, if a system of $\mathrm{OD} / \mathrm{PD}$ equations is written in the form:

$$
\Phi^{\tau} \equiv a_{k}^{\tau \mu} y_{\mu}^{k}=0
$$

with coefficients $a_{k}^{\tau \mu} \in K$, we may introduce the free differential module $D y=D y^{1}+\cdots+D y^{m} \simeq D^{m}$ and consider the differential submodule $I=D \Phi \subset D y$ which is usually called the module of equations, both with the differential module $M=D y / D \Phi$ or $D$-module and we may set $M={ }_{D} M$ if we want to specify the ring of differential operators. The work of Macaulay only 
covers the case $m=1$ with $K$ replaced by $k \subseteq c s t(K)$. Again, we may introduce the formal prolongation with respect to $d_{i}$ by setting:

$$
d_{i} \Phi^{\tau} \equiv a_{k}^{\tau \mu} y_{\mu+1_{i}}^{k}+\left(\partial_{i} a_{k}^{\tau \mu}\right) y_{\mu}^{k}
$$

in order to induce maps $d_{i}: M \rightarrow M: \bar{y}_{\mu}^{k} \rightarrow \bar{y}_{\mu+1_{i}}^{k}$ if we use to denote the residue $D y \rightarrow M: y^{k} \rightarrow \bar{y}^{k}$ by a bar as in algebraic geometry. However, for simplicity, we shall not write down the bar when the background will indicate clearly if we are in $D y$ or in $M$.

As a byproduct, the differential modules we shall consider will always be finitely generated $(k=1, \cdots, m<\infty)$ and finitely presented $(\tau=1, \cdots, p<\infty)$. Equivalently, introducing the matrix of operators $\mathcal{D}=\left(a_{k}^{\tau \mu} d_{\mu}\right)$ with $m$ columns and $p$ rows, we may introduce the morphism $D^{p} \stackrel{\mathcal{D}}{\longrightarrow} D^{m}:\left(P_{\tau}\right) \rightarrow\left(P_{\tau} \Phi^{\tau}\right): P \rightarrow P \Phi=P \mathcal{D}$ over $D$ by acting with $D$ on the left of these row vectors while acting with $\mathcal{D}$ on the right of these row vectors and the presentation of $M$ is defined by the exact cokernel sequence $D^{p} \rightarrow D^{m} \rightarrow M \rightarrow 0$. It is essential to notice that the presentation only depends on $K, D$ and $\Phi$ or $\mathcal{D}$, that is to say never refers to the concept of (explicit or formal) solutions. It is at this moment that we have to take into account the results of the previous section in order to understand that certain presentations will be much better than others, in particular to establish a link with formal integrability and involution.

DEFINITION 2.7: It follows from its definition that $M$ can be endowed with a quotient filtration obtained from that of $D^{m}$ which is defined by the order of the jet coordinates $y_{q}$ in $D_{q} y$. We have therefore the inductive limit $0 \subseteq M_{0} \subseteq M_{1} \subseteq \cdots \subseteq M_{q} \subseteq \cdots \subseteq M_{\infty}=M$ with $d_{i} M_{q} \subseteq M_{q+1}$ and $M=D M_{q}$ for $q \gg 0$ with prolongations $D_{r} M_{q} \subseteq M_{q+r}, \forall q, r \geq 0$. We shall set $\operatorname{gr}\left(M_{q}\right)=G_{q}=M_{q} / M_{q-1}$ and $\operatorname{gr}(M)=G=\oplus_{q} G_{q}$.

Having in mind that $K$ is a left $D$-module for the action $(D, K) \rightarrow K:\left(d_{i}, a\right) \rightarrow \partial_{i} a$ and that $D$ is a bimodule over itself, we have only two possible constructions:

DEFINITION 2.8: We define the system $R=\operatorname{hom}_{K}(M, K)=M^{*}$ and set $R_{q}=\operatorname{hom}_{K}\left(M_{q}, K\right)=M_{q}^{*}$ as the system of order $q$. We have the projective limit $R=R_{\infty} \rightarrow \cdots \rightarrow R_{q} \rightarrow \cdots \rightarrow R_{1} \rightarrow R_{0}$. It follows that $f_{q} \in R_{q}: y_{\mu}^{k} \rightarrow f_{\mu}^{k} \in K$ with $a_{k}^{\tau \mu} f_{\mu}^{k}=0$ defines a section at order $q$ and we may set $f_{\infty}=f \in R$ for a section of $R$. For a ground field of constants $k$, this definition has of course to do with the concept of a formal power series solution. However, for an arbitrary differential field $K$, the main novelty of this new approach is that such a definition has nothing to do with the concept of a formal power series solution (care) as illustrated in ([39]).

DEFINITION 2.9: We may define the right differential module $\operatorname{ext}^{0}(M)=\operatorname{hom}_{D}(M, D)$.

PROPOSITION 2.10: When $M$ is a left $D$-module, then $R$ is also a left $D$-module.

Proof. As $D$ is generated by $K$ and $T$ as we already said, let us define: 


$$
\begin{gathered}
(a f)(m)=a f(m), \quad \forall a \in K, \forall m \in M \\
(\xi f)(m)=\xi f(m)-f(\xi m), \quad \forall \xi=a^{i} d_{i} \in T, \forall m \in M
\end{gathered}
$$

In the operator sense, it is easy to check that $d_{i} a=a d_{i}+\partial_{i} a$ and that $\xi \eta-\eta \xi=[\xi, \eta]$ is the standard bracket of vector fields. We finally get $\left(d_{i} f\right)_{\mu}^{k}=\left(d_{i} f\right)\left(y_{\mu}^{k}\right)=\partial_{i} f_{\mu}^{k}-f_{\mu+1_{i}}^{k}$ and thus recover exactly the Spencer operator though this is not evident at all. We also get

$\left(d_{i} d_{j} f\right)_{\mu}^{k}=\partial_{i j} f_{\mu}^{k}-\partial_{i} f_{\mu+1_{j}}^{k}-\partial_{j} f_{\mu+1_{i}}^{k}+f_{\mu+1_{i}+1_{j}}^{k} \Rightarrow d_{i} d_{j}=d_{j} d_{i}, \forall i, j=1, \cdots, n \quad$ and thus $d_{i} R_{q+1} \subseteq R_{q} \Rightarrow d_{i} R \subset R$ induces a well defined operator

$R \rightarrow T^{*} \otimes R: f \rightarrow d x^{i} \otimes d_{i} f$. This result has been discovered (up to sign) by Macaulay in 1916 ([21]). For more details on the Spencer operator and its applications, the reader may look at ([14] [40] [41] [42]).

Q.E.D.

DEFINITION 2.11: $t_{r}(M)$ is the greatest differential submodule of $M$ having codimension $>r$.

PROPOSITION 2.12: $c d(M)=c d(V)=r \Leftrightarrow \alpha_{q}^{n-r} \neq 0$, $\alpha_{q}^{n-r+1}=\cdots=\alpha_{q}^{n}=0 \Leftrightarrow t_{r}(M) \neq M, t_{r-1}(M)=\cdots=t_{0}(M)=t(M)=M$ and this intrinsic result can be most easily checked by using the standard or reduced Spencer form of the system defining $M$.

We are now in a good position for defining and studying purity for differential modules.

DEFINITION 2.13: $M$ is $r$-pure $\Leftrightarrow t_{r}(M)=0, t_{r-1}(M)=M \Leftrightarrow c d(D m)=r$, $\forall m \in M$. More generally, $M$ is pure if it is $r$-pure for a certain $0 \leq r \leq n$. In particular, $M$ is 0 -pure if $t(M)=0$ and, if $c d(M)=r$ but $M$ is not $r$-pure, we may call $M / t_{r}(M)$ the pure part of $M$. It follows that $t_{r-1}(M) / t_{r}(M)$ is equal to zero or is $r$-pure (see the picture in [3], p. 545). When $M=t_{n-1}(M)$ is $n$-pure, its defining system is a finite dimensional vector space over $K$ with a symbol of finite type, that is when $g_{q}=0$ is (trivially) involutive. Finally, when $t_{r-1}(M)=t_{r}(M)$, we shall say that there is a "gap" in the purity filtration:

$$
0=t_{n}(M) \subseteq t_{n-1}(M) \subseteq \cdots \subseteq t_{1}(M) \subseteq t_{0}(M)=t(M) \subseteq M
$$

PROPOSITION 2.14: $t_{r}(M)$ does not depend on the presentation or on the filtration of $M$.

EXAMPLE 2.15: If $K=\mathbb{Q}$ and $M$ is defined by the involutive system $y_{33}=0, y_{23}=0, y_{13}=0$, then $z=y_{3} \quad$ satisfies $\quad d_{3} z=0, d_{2} z=0, d_{1} z=0 \quad$ and $c d(D z)=3$ while $z^{\prime}=y_{2}$ only satisfies $d_{3} z^{\prime}=0$ and $c d\left(D z^{\prime}\right)=1$. We have the purity filtration $0=t_{3}(M) \subset t_{2}(M)=t_{1}(M) \subset t_{0}(M)=t(M)=M$ with one gap and two strict inclusions.

We now recall the definition of the extension modules $\operatorname{ext}_{D}^{i}(M, D)$ that we shall simply denote by $\operatorname{ext}^{i}(M)$ and the way to use their dimension or codimension. We point out once more that these numbers can be most easily obtained by bringing the underlying systems to involution in order to get informations on $M$ from informations on $G$. We divide the procedure into four steps 
that can be achieved by means of computer algebra ([11] [12]):

- STEP 1: Construct a free resolution of $M$, say:

$$
\cdots \rightarrow F_{i} \rightarrow \cdots \rightarrow F_{1} \rightarrow F_{0} \rightarrow M \rightarrow 0
$$

- STEP 2: Suppress $M$ in order to obtain the deleted sequence:

$$
\cdots \rightarrow F_{i} \rightarrow \cdots \rightarrow F_{1} \rightarrow F_{0} \rightarrow 0
$$

- STEP 3: Apply $\operatorname{hom}_{D}(\bullet, D)$ in order to obtain the dual sequence heading backwards:

$$
\cdots \leftarrow \operatorname{hom}_{D}\left(F_{i}, D\right) \leftarrow \cdots \leftarrow \operatorname{hom}_{D}\left(F_{1}, D\right) \leftarrow \operatorname{hom}_{D}\left(F_{0}, D\right) \leftarrow 0
$$

- STEP 4: Define $\operatorname{ext}^{i}(M)$ to be the cohomology at $\operatorname{hom}_{D}\left(F_{i}, D\right)$ of the dual sequence in such a way that $\operatorname{ext}^{0}(M)=\operatorname{hom}_{D}(M, D)$.

The following nested chain of difficult propositions and theorems can be obtained, even in the non-commutative case, by combining the use of extension modules and bidualizing complexes in the framework of algebraic analysis. The main difficulty is to obtain first these results for the graded module $G=\operatorname{gr}(M)$ by using techniques from commutative algebra before extending them to the filtered module $M$ as in ([1] [2] [3] [4] [24] [25] [26] [27] [37] [42]).

THEOREM 2.16: The extension modules do not depend on the resolution of $M$ used.

PROPOSITION 2.17: Applying $\operatorname{hom}_{D}(\bullet, D)$ provides right $D$-modules that can be transformed to left $D$-modules by means of the side changing functor and vice-versa. Namely, if $N_{D}$ is a right $D$-module, then ${ }_{D} N=\wedge^{n} T \otimes_{K} N_{D}$ is the converted left $D$-module while, if $N={ }_{D} N$ is a left $D$-module, then $N_{D}=\wedge^{n} T^{*} \otimes_{K} N$ is the converted right $D$-module.

PROPOSITION 2.18: Instead of using $\operatorname{hom}_{D}(\bullet, D)$ and the side changing functor in the module framework, we may use $a d$ in the operator framework. Namely, to any operator $\mathcal{D}: E \rightarrow F$ we may associate the formal adjoint $\operatorname{ad}(\mathcal{D}): \wedge^{n} T^{*} \otimes F^{*} \rightarrow \wedge^{n} T^{*} \otimes E^{*}$ with the useful though striking relation $r k_{D}(\operatorname{ad}(\mathcal{D}))=r k_{D}(\mathcal{D})$.

PROPOSITION 2.19: $\operatorname{ext}^{i}(M)$ is a torsion module $\forall 1 \leq i \leq n$ but $\operatorname{ext}^{0}(M)=\operatorname{hom}_{D}(M, D)$ may not be a torsion module.

EXAMPLE 2.20: When $M$ is a torsion module, we have $\operatorname{hom}_{D}(M, D)=0$. Indeed, if $m \in M$, we may find $0 \neq P \in D$ such that $P m=0$. Hence, if $f \in \operatorname{hom}_{D}(M, D)$, we have $P f(m)=f(P m)=f(0)=0$ in $D$ and thus $f(m)=0, \forall m \in M$, that is to say $f=0$ because $D$ is an integral domain. When $n=3$ and the torsion-free module $M$ is defined by the formally surjective div operator, the formal adjoint of div is - grad which defines a torsion module. Also, when $n=1$ as in classical control theory, a controllable system with coefficients in a differential field allows to define a torsion-free module $M$ which is free in that case because a finitely generated module over a principal ideal domain is free if and only if it is torsion-free and $\operatorname{hom}_{D}(M, D)$ is thus also a free module.

THEOREM 2.21: $\operatorname{ext}^{i}(M)=0, \forall i<c d(M)$ and $\forall i \geq n+1$. 
THEOREM 2.22: $c d\left(\operatorname{ext}^{i}(M)\right) \geq i$.

THEOREM 2.23: $c d(M) \geq r \Leftrightarrow \operatorname{ext}^{i}(M)=0, \forall i<r$.

PROPOSITION 2.24: $\quad c d(M)=r \Rightarrow c d\left(\operatorname{ext}^{r}(M)\right)=r \quad$ and $\quad \operatorname{ext}^{r}(M)$ is $r$-pure.

PROPOSITION 2.25: $\operatorname{ext}^{r}\left(\operatorname{ext}^{r}(M)\right)$ is equal to 0 or is $r$-pure, $\forall 0 \leq r \leq n$.

PROPOSITION 2.26: If we set $t_{-1}(M)=M$, there are exact sequences $\forall 0 \leq r \leq n$ :

$$
0 \rightarrow t_{r}(M) \rightarrow t_{r-1}(M) \rightarrow \operatorname{ext}^{r}\left(\operatorname{ext}^{r}(M)\right)
$$

THEOREM 2.27: If $c d(M)=r$, then $M$ is $r$-pure if and only if there is a monomorphism $0 \rightarrow M \rightarrow \operatorname{ext}^{r}\left(\operatorname{ext}^{r}(M)\right)$ of left differential modules.

THEOREM 2.28: $M$ is pure $\Leftrightarrow \operatorname{ext}^{s}\left(\operatorname{ext}^{s}(M)\right)=0, \forall s \neq c d(M)$.

COROLLARY 2.29: If $M$ is $r$-pure with $r \geq 1$, then it can be embedded into a differential module $L$ having a free resolution with only $r$ operators.

The previous theorems are known to characterize purity but it is however evident that they are not very useful in actual practice. For more details on these two results which are absolutely out of the scope of this paper, see ([2], pp. 490-491) and ([3], p. 547). Proposition 3.24 and Theorem 3.25 come from the Cohen-Macaulay property of $M$, namely $c d(M)=g(M)=\inf \left\{i \mid \operatorname{ext}^{i}(M) \neq 0\right\}$ where $g(M)$ is called the grade of $M$ (see [2] and [3] [4] for more details).

THEOREM 2.30: When $M$ is $r$-pure, the characteristic ideal is thus unmixed, that is a finite intersection of prime ideals having the same codimension $r$ and the characteristic set is equidimensional, that is the union of irreducible algebraic varieties having the same codimension $r$.

In 2012 we have provided a new effective test for checking purity while using the involutivity of the Spencer form with four steps as follows ([4]):

- STEP 1: Compute the involutive Spencer form of the system and the number $r$ of full classes.

- STEP 2: Select only the equations of class 1 to $d(M)=n-r$ of this Spencer form which are making an involutive system over $K\left[d_{1}, \cdots, d_{(n-r)}\right]$.

- STEP 3: Using differential biduality for such a system, check if it defines a torsion-free module $M_{(n-r)}$ and work out a parametrization.

- STEP 4: Substitute the above parametrization in the remaning equations of class $n-r+1, \cdots, n$ of the Spencer form in order to get a system of PD equations which provides the parametrizing module $L$ in such a way that $M \subseteq L$ and $L$ has a resolution with $r$ operators.

THEOREM 2.31: As purity is an intrinsic property, we may work with an involutive Spencer form and $M$ is $r$-pure if the classes $n-r+1, \cdots, n$ are full and the module $M_{(n-r)}$ defined by the equations of class $1+\cdots+$ class $(n-r)$ is torsion-free. Hence $M$ is 0 -pure if it is torsion-free.

We shall now illustrate and apply this new procedure in the next two sections.

\section{Motivating Examples}

EXAMPLE 3.1: With $n=3, m=1$ and $K=\mathbb{Q}$, let us consider the following 
polynomial ideal:

$$
\mathfrak{a}=\left(\left(\chi_{3}\right)^{2}, \chi_{2} \chi_{3}-\chi_{1} \chi_{3},\left(\chi_{2}\right)^{2}-\chi_{1} \chi_{2}\right) \subset K\left[\chi_{1}, \chi_{2}, \chi_{3}\right]=K[\chi]
$$

We shall discover that it is not evident to prove that it is an unmixed polynomial ideal and that the corresponding differential module is 1-pure.

The first result is provided by the existence of the primary decomposition obtained from the two existing factorizations ([23]):

$$
\mathfrak{a}=\left(\left(\chi_{3}\right)^{2}, \chi_{2}-\chi_{1}\right) \cap\left(\chi_{3}, \chi_{2}\right)=\mathfrak{q}^{\prime} \cap \mathfrak{q}^{\prime \prime}
$$

Taking the respective radical ideals, we get the prime decomposition:

$$
\operatorname{rad}(\mathfrak{a})=\left(\chi_{3}, \chi_{2}-\chi_{1}\right) \cap\left(\chi_{3}, \chi_{2}\right)=\mathfrak{p}^{\prime} \cap \mathfrak{p}^{\prime \prime}=\operatorname{rad}\left(\mathfrak{q}^{\prime}\right) \cap \operatorname{rad}\left(\mathfrak{q}^{\prime \prime}\right)
$$

The corresponding involutive system is:

$$
\left\{\begin{array}{l}
y_{33}=0 \\
y_{23}-y_{13}=0 \\
y_{22}-y_{12}=0
\end{array} \begin{array}{lll}
1 & 2 & 3 \\
1 & 2 & \bullet \\
1 & 2 & \bullet
\end{array}\right.
$$

with characters $\quad \alpha_{2}^{3}=1-1=0 \quad, \quad \alpha_{2}^{2}=2-2=0 \quad, \quad \alpha_{2}^{1}=3-0=3 \quad$ and $\operatorname{dim}\left(g_{2}\right)=\sum \alpha=3$.

Setting $\left(z^{1}=y, z^{2}=y_{1}, z^{3}=y_{2}, z^{4}=y_{3}\right)$, we obtain the involutive first order Spencer form:

$$
\left\{\begin{array}{l}
z_{3}^{4}=0, z_{3}^{3}-z_{1}^{4}=0, z_{3}^{2}-z_{1}^{4}=0, z_{3}^{1}-z^{4}=0 \\
z_{2}^{4}-z_{1}^{4}=0, z_{2}^{3}-z_{1}^{3}=0, z_{2}^{2}-z_{1}^{3}=0, z_{2}^{1}-z^{3}=0 \\
z_{1}^{1}-z^{2}=0
\end{array} \quad \begin{array}{lll}
1 & 2 & 3 \\
1 & 2 & \bullet \\
1 & \bullet & \bullet
\end{array}\right.
$$

with new characters $\alpha_{1}^{3}=4-4=0, \alpha_{1}^{3}=4-4=0, \alpha_{1}^{1}=4-1=3$ and similarly $\operatorname{dim}\left(g_{1}\right)=\sum \alpha=3$. Both class 3 and class 2 are full while class 1 is defining a torsion-free module $M_{(1)}$ over $K\left[d_{1}\right]$ by means of a trivially involutive system of class 1 . Hence the differential module $M$ is such that $c d(M)=2$ and is 1-pure because it is 1-pure in this presentation.

Suppressing the bar for the various residues, we are ready to exhibit the relative parametrization defining the parametrization module $L$ because we may choose the 3 potentials $\left(z^{1}=y, z^{3}, z^{4}\right)$ while taking into account that $z^{2}=y_{1}=d_{1} y$ :

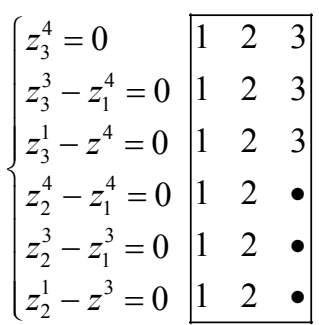

Both $\left(y, z^{3}, z^{4}\right)$ are torsion elements and we can eliminate $\left(z^{3}, z^{4}\right)$ in order to find the desired system that must be satisfied by $y$ which is showing the inclusion $M \subset L$ but we have indeed $M=L$ because $z^{3}=y_{2}, z^{4}=y_{3}$. It follows that $M$ admits a free resolution with only 2 operators, a result following at once 
from the last Janet tabular, contrary to the previous one.

The reader may treat similarly the example $\mathfrak{a}=\left(\chi_{1}, \chi_{2}\right) \cap\left(\chi_{3}, \chi_{4}\right)$ and look at ([39]) for details. (Hint: use the involutive system $y_{44}+y_{14}=0, y_{34}+y_{13}=0$, $\left.y_{33}+y_{23}=0, y_{24}-y_{13}=0\right)$.

EXAMPLE 3.2: With $n=3, m=1, q=2, K=\mathbb{Q}, D=K\left[d_{1}, d_{2}, d_{3}\right]$, let us consider the differential module $M$ defined by the second order system $P y \equiv y_{33}=0, Q y \equiv y_{13}-y_{2}=0$ first considered by Macaulay in 1916 ([19] [21]). We shall prove that $M$ is 2-pure through the inclusion $0 \rightarrow M \rightarrow \operatorname{ext}^{2}\left(\operatorname{ext}^{2}(M)\right)$ directly and by finding out a relative parametrization, a result highly not evident at first sight.

First of all, in order to find out the codimension $c d(M)=2$, we have to consider the equivalent involutive system:

$$
\left\{\begin{array}{l|l}
\Phi^{4} \equiv y_{33}=u \\
\Phi^{3} \equiv y_{23}=d_{1} u-d_{3} v \\
\Phi^{2} \equiv y_{22}=d_{11} u-d_{13} v-d_{2} v \\
\Phi^{1} \equiv y_{13}-y_{2}=v
\end{array}\right.
$$

The Janet tabular on the right allows at once to compute the characters $\alpha_{2}^{3}=0$, $\alpha_{2}^{2}=0, \alpha_{2}^{1}=\alpha=3-1=2$ and to construct the following strictly exact sequence of differential modules:

$$
0 \rightarrow D \rightarrow D^{4} \rightarrow D^{4} \rightarrow D \stackrel{p}{\longrightarrow} M \rightarrow 0
$$

Also, we have

$$
\operatorname{rad}(\mathfrak{a})=\operatorname{rad}\left(\left(\chi_{3}\right)^{2}, \chi_{2} \chi_{3},\left(\chi_{2}\right)^{2}, \chi_{1} \chi_{3}\right)=\left(\chi_{3}, \chi_{2}\right)=\mathfrak{p} \Rightarrow \operatorname{dim}(V)=1 .
$$

As the classes 3 and 2 are full, it follows that $d(M)=d(D y)=1 \Rightarrow c d(M)=n-1=2$ if we denote simply by $y$ the canonical residue $\bar{y}$ of $y$ after identifying $D$ with $D y$. We have constructed explicitly in ([29]) a finite length resolution of $N=\operatorname{ext}^{2}(M)$ by pointing out that $N$ does not depend on the resolution of $M$ used and one can refer to the single compatibility condition (CC) $P \circ Q y-Q \circ P y=0$ for the initial system in the exact sequence made by second order operators:

$$
0 \rightarrow D \stackrel{\mathcal{D}_{1}}{\longrightarrow} D^{2} \stackrel{\mathcal{D}}{\longrightarrow} D \stackrel{p}{\longrightarrow} M \rightarrow 0
$$

Indeed, introducing differential duality through the functor $\operatorname{hom}_{D}(\bullet, D)$ and the respective adjoint operators, we may define the torsion left differential module $N$ by the long exact sequence:

$$
0 \leftarrow N \stackrel{q}{\longleftarrow} D \stackrel{a d\left(\mathcal{D}_{1}\right)}{\longleftarrow} D^{2} \stackrel{a d(\mathcal{D})}{\longrightarrow} D \leftarrow 0
$$

showing that $r k_{D}(M)=0 \Rightarrow r k_{D}(N)=1-2+1=0$ because of the additivity property of the differential rank and the vanishing of the Euler-Poincaré characteristic of the full sequence. It follows that $M=\operatorname{ext}^{2}(N)=\operatorname{ext}^{2}\left(\operatorname{ext}^{2}(M)\right)$.

Similarly, using certain parametric jet variables as new unknowns, we may set $z^{1}=y, \quad z^{2}=y_{1}, z^{3}=y_{2}, \quad z^{4}=y_{3}$ in order to obtain the following involutive first order system with no zero order equation: 


$$
\begin{cases}\text { class } 3 & d_{3} z^{1}-z^{4}=0, d_{3} z^{2}-z^{3}=0, d_{3} z^{3}=0, d_{3} z^{4}=0 \\ \text { class 2 } & d_{2} z^{1}-z^{3}=0, d_{2} z^{2}-d_{1} z^{3}=0, d_{2} z^{3}=0, d_{2} z^{4}=0 \\ \text { class 1 } & d_{1} z^{1}-z^{2}=0, d_{1} z^{4}-z^{3}=0\end{cases}
$$

where we have separated the classes while using standard computer algebra notations this time instead of the jet notations used in the previous example. Contrary to what could be believed, this operator does not describe the Spencer sequence that could be obtained from the previous Janet sequence but we can use it exactly like a Janet sequence or exactly like a Spencer sequence. We obtain therefore a long strictly exact sequence of differential modules with only first order operators while replacing $D y$ by $D z=D z^{1}+D z^{2}+D z^{3}+D z^{4}$ as follows:

$$
0 \rightarrow D^{2} \longrightarrow D^{8} \longrightarrow D^{10} \longrightarrow D^{4} \rightarrow M \rightarrow 0
$$

and we still have the vanishing Euler-Poincaré characteristic $2-8+10-4=0$.

The differential module $M_{1}$ is defined over $K\left[d_{1}\right]$ by the two PD equations of class 1 and is easily seen to be torsion-free with the two potentials $\left(z^{1}=y, z^{4}\right)$. Substituting into the PD equations of class 2 and 3 , we obtain the generating differential constraints:

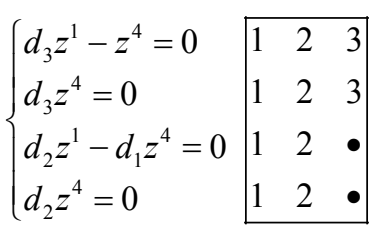

They define the parametrization module $L$ and the inclusion $M \subseteq L$ is obtained by eliminating $z^{4}$ but we have indeed $M=L$ because $z^{4}=d_{3} y$.

EXAMPLE 3.3: We have provided in ([4], Example 4.2) a case leading to a strict inclusion $M \subset L$ that we revisit now totally in this new framework. With $K=\mathbb{Q}, m=1, n=4, q=2$, let us study the 2-pure differential module $M$ defined by the involutive second order system:

$$
\left\{\begin{array}{l}
y_{44}=0 \\
y_{34}=0 \\
y_{33}=0 \\
y_{24}-y_{13}=0
\end{array} \quad \begin{array}{llll}
1 & 2 & 3 & 4 \\
1 & 2 & 3 & \bullet \\
1 & 2 & 3 & \bullet \\
1 & 2 & \bullet & \bullet
\end{array}\right.
$$

From the Janet tabular we may construct at once the Janet sequence:

$$
0 \rightarrow \Theta \rightarrow 1 \stackrel{\mathcal{D}}{\longrightarrow} 4 \stackrel{\mathcal{D}_{1}}{\longrightarrow} 4 \stackrel{\mathcal{D}_{2}}{\longrightarrow} 1 \rightarrow 0
$$

where $\mathcal{D}_{1}$ is defined by the involutive system:

$$
\left\{\begin{array}{l}
d_{4}\left(y_{34}\right)-d_{3}\left(y_{44}\right)=0 \\
d_{4}\left(y_{33}\right)-d_{3}\left(y_{34}\right)=0 \\
d_{4}\left(y_{24}-y_{13}\right)-d_{2}\left(y_{44}\right)+d_{1}\left(y_{34}\right)=0 \\
d_{3}\left(y_{24}-y_{13}\right)-d_{2}\left(y_{34}\right)+d_{1}\left(y_{33}\right)=0
\end{array} \begin{array}{llll}
1 & 2 & 3 & 4 \\
1 & 2 & 3 & 4 \\
1 & 2 & 3 & 4 \\
1 & 2 & 3 & \bullet
\end{array}\right.
$$

and so on. We have therefore a free resolution of $M$ with 3 operators: 


$$
0 \rightarrow D \rightarrow D^{4} \rightarrow D^{4} \rightarrow D \rightarrow M \rightarrow 0
$$

and thus discover that $p d(M) \leq 3$.

However, we have

$$
\operatorname{rad}\left(\left(\chi_{4}\right)^{2}, \chi_{3} \chi_{4},\left(\chi_{3}\right)^{2}, \chi_{2} \chi_{4}-\chi_{1} \chi_{3}\right)=\left(\chi_{4}, \chi_{3}\right)=\mathfrak{p} \Rightarrow \operatorname{dim}(V)=1 .
$$

Let us transform the initial second order involutive system for $y$ into a first order involutive system for $\left(z^{1}=y, z^{2}=y_{1}, z^{3}=y_{2}, z^{4}=y_{3}, z^{5}=y_{4}\right)$ as follows:

$$
\left\{\begin{array}{l}
d_{4} z^{1}-z^{5}=0, d_{4} z^{2}-d_{1} z^{5}=0, d_{4} z^{3}-d_{1} z^{4}=0, d_{4} z^{4}=0, d_{4} z^{5}=0 \\
d_{3} z^{1}-z^{4}=0, d_{3} z^{2}-d_{1} z^{4}=0, d_{3} z^{3}-d_{2} z^{4}=0, d_{3} z^{4}=0, d_{3} z^{5}=0 \\
d_{2} z^{1}-z^{3}=0, d_{2} z^{2}-d_{1} z^{3}=0, d_{2} z^{5}-d_{1} z^{4}=0 \\
d_{1} z^{1}-z^{2}=0
\end{array} \mid \begin{array}{llll}
1 & 2 & 3 & 4 \\
1 & 2 & \bullet & \bullet \\
1 & \bullet & \bullet & \bullet
\end{array}\right.
$$

with five equations of full class 4 , five equations of full class 3 , three equations of class 2 and finally one equation of class 1 . The equations of classes 2 and 1 are providing an involutive system over $\mathbb{Q}\left[d_{1}, d_{2}\right]$ defining a torsion-free module $M_{(2)}$ that can be parametrized by setting $z^{1}=y, z^{2}=d_{1} y, z^{3}=d_{2} y, z^{4}=d_{2} z, z^{5}=d_{1} z$ with only 2 arbitrary potentials $(y, z)$. Substituting in the other equations of classes 3 and 4 , we finally discover that $L$ is defined by the involutive system describing the relative parametrization:

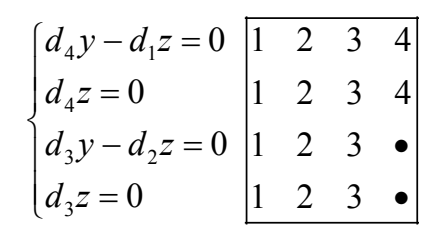

We have the strict inclusion $M \subset L$ obtained by eliminating $z$ because now $z \notin D y \quad$ if we take the residue or, equivalently, the residue of $z$ does not belong to $M$. The differential module $L$ defined by the above system is therefore 2-pure with a strict inclusion $M \subset L$ and admits a free resolution with only 2 operators according to its Janet tabular.

EXAMPLE 3.4: (Contact structure) With $n=m=3$ and $K=\mathbb{Q}\left(x^{1}, x^{2}, x^{3}\right)$ let us introduce the so-called contact 1-form $\alpha=d x^{1}-x^{3} d x^{2}$ and consider the first order system of infinitesimal Lie equations obtained by eliminating the contact factor $\rho$ from the equations $L(\xi) \alpha=\rho \alpha$. We let the reader check that he will obtain only the two equations $\Phi^{1}=0, \Phi^{2}=0$ which is nevertheless neither formally integrable nor even involutive. Using crossed derivatives one obtains the involutive system:

$$
\left\{\begin{array}{l|}
\Phi^{3} \equiv \partial_{3} \xi^{3}+\partial_{2} \xi^{2}+2 x^{3} \partial_{1} \xi^{2}=0 \\
\Phi^{2} \equiv \partial_{3} \xi^{1}-x^{3} \partial_{3} \xi^{2}=0 \\
\Phi^{1} \equiv \partial_{2} \xi^{1}-x^{3} \partial_{2} \xi^{2}+x^{3} \partial_{1} \xi^{1}-\left(x^{3}\right)^{2} \partial_{1} \xi^{2}-\xi^{3}=0
\end{array} \mid \begin{array}{lll}
1 & 2 & 3 \\
1 & 2 & 3 \\
1 & 2 & \bullet
\end{array}\right.
$$

with the unique CC $\Psi \equiv \partial_{3} \Phi^{1}-\partial_{2} \Phi^{2}-x^{3} \partial_{1} \Phi^{2}+\Phi^{3}=0$. The following injective absolute parametrization is well known and we let the reader find it by using differential double duality: 


$$
\phi-x^{3} \partial_{3} \phi=\xi^{1},-\partial_{3} \phi=\xi^{2}, \partial_{2} \phi+x^{3} \partial_{1} \phi=\xi^{3} \Rightarrow \xi^{1}-x^{3} \xi^{2}=\phi
$$

We obtain the Janet sequence

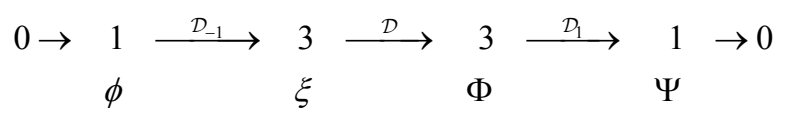

with formally exact adjoint sequence:

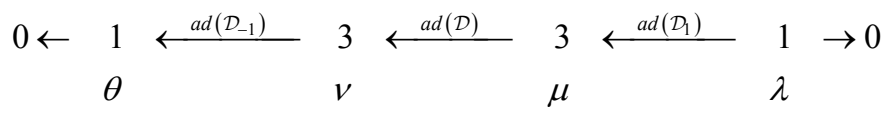

and the resolution of the trivially torsion-free module $M \simeq D$ :

$$
0 \rightarrow D \rightarrow D^{3} \rightarrow D^{3} \rightarrow M \rightarrow 0
$$

which splits totally because it is made with free and thus projective modules.

EXAMPLE 3.5: (Unimodular contact structure) With $n=m=3$ and $K=\mathbb{Q}\left(x^{1}, x^{2}, x^{3}\right)$ let us introduce the 1 -form $\omega=d x^{1}-x^{3} d x^{2}$ used as a geometric object and consider the first order system of infinitesimal Lie equations from the equations $L(\xi) \omega=0$. One obtains the system using jet notations:

$$
\xi_{3}^{1}-x^{3} \xi_{3}^{2}=0, \xi_{2}^{1}-x^{3} \xi_{2}^{2}-\xi^{3}=0, \xi_{1}^{1}-x^{3} \xi_{1}^{2}=0
$$

We let the reader prove that these three $\mathrm{PD}$ equations are differentially independent and we obtain the free resolution of $M$ :

$$
0 \rightarrow D^{3} \stackrel{\mathcal{D}}{\longrightarrow} D^{3} \rightarrow M \rightarrow 0
$$

and its adjoint sequence is:

$$
0 \leftarrow N \leftarrow D^{3} \stackrel{a d(\mathcal{D})}{\longleftarrow} D^{3} \leftarrow 0
$$

because $r k_{D}(M)=r k_{D}(N)=3-3=0$, that is both $M$ and $N$ are torsion modules with $N=\operatorname{ext}^{1}(M) \Rightarrow M=\operatorname{ext}^{1}(N)=\operatorname{ext}^{1}\left(\operatorname{ext}^{1}(M)\right)$ and $M$ is surely 1-pure. However, this system is not formally integrable, as it can be checked directly through crossed derivatives or by noticing that $\mathcal{L}(\xi) d \omega=0$ with $d \omega=d x^{2} \wedge d x^{3}$ and $\mathcal{L}(\xi)(\omega \wedge d \omega)=0$ with $\omega \wedge d \omega=d x^{1} \wedge d x^{2} \wedge d x^{3}$. Hence, we have to add the 3 first order equations:

$$
\xi_{2}^{2}+\xi_{3}^{3}=0, \xi_{1}^{3}=0, \xi_{1}^{2}=0 \Rightarrow \xi_{1}^{1}=0
$$

Exchanging $x^{1}$ and $x^{3}$, we obtain the equivalent involutive system in $\delta$ -regular coordinates:

$$
\left\{\begin{array}{l|l}
\xi_{3}^{3}=0 & \\
\xi_{3}^{2}=0 \\
\xi_{3}^{1}=0 \\
\xi_{2}^{2}+\xi_{1}^{3}=0 \\
\xi_{2}^{1}+x^{1} \xi_{1}^{3}-\xi^{3}=0 \\
\xi_{1}^{1}-x^{1} \xi_{1}^{2}=0
\end{array} \mid \begin{array}{lll}
1 & 2 & 3 \\
1 & 2 & 3 \\
1 & 2 & 3 \\
1 & 2 & \bullet \\
1 & 2 & \bullet \\
1 & \bullet & \bullet
\end{array}\right.
$$

The differential module $M_{(2)}$ over $K\left[d_{1}, d_{2}\right]$ is defined by the three bottom equations. Setting now $\phi=\xi^{1}-x^{1} \xi^{2}$, we deduce from the last bottom equation that $\xi^{2}=-d_{1} \phi$ and thus $\xi^{1}=\phi-x^{1} d_{1} \phi$. Finally, substituting in the equation 
before the last, we get $\xi^{3}=d_{2} \phi$. We have thus obtained an injective parametrization of $M_{(2)}$ which is therefore torsion-free and $M$ is 2-pure in a coherent way. Substituting into the three upper equations, we obtain the desired relative parametrization by adding the differential constraint $d_{3} \phi=0$. Coming back to the original coordinates, we obtain the relative parametrization:

$$
\phi-x^{3} d_{3} \phi=\xi^{1},-d_{3} \phi=\xi^{2}, d_{2} \phi=\xi^{3} \text { with } d_{1} \phi=0 \Rightarrow d_{2} \phi+x^{3} d_{1} \phi=\xi^{3}
$$

which is thus strikingly obtained from the previous contact parametrization by adding the only differential constraint $d_{1} \phi=0$ obtained by substituting it in the new system of Lie equations.

\section{Applications}

Before studying applications to mathematical physics, we shall start with an example describing in an explicit way the Janet and Spencer sequences used thereafter, both with their link, namely the relations existing between the dimensions of the respective Janet and Spencer bundles.

EXAMPLE 4.1: When $n=m=2, q=2, \omega$ is the Euclidean metric of $X=\mathbb{R}^{2}$ with Christoffel symbols $\gamma$ and metric density $\tilde{\omega}=\omega / \sqrt{\operatorname{det}(\omega)}$, we consider the two involutive systems of linear infinitesimal Lie equations $R_{2} \subset \tilde{R}_{2} \subset J_{2}(T)$ respectively defined by $\{L(\xi) \omega=0, L(\xi) \gamma=0\}$ and $\{L(\xi) \tilde{\omega}=0, L(\xi) \gamma=0\}$. We have $g_{2}=\tilde{g}_{2}=0$ and construct the following successive commutative and exact diagrams followed by the corresponding dimensional diagrams that are used in order to construct effectively the respective Janet and Spencer differential sequences while comparing them.

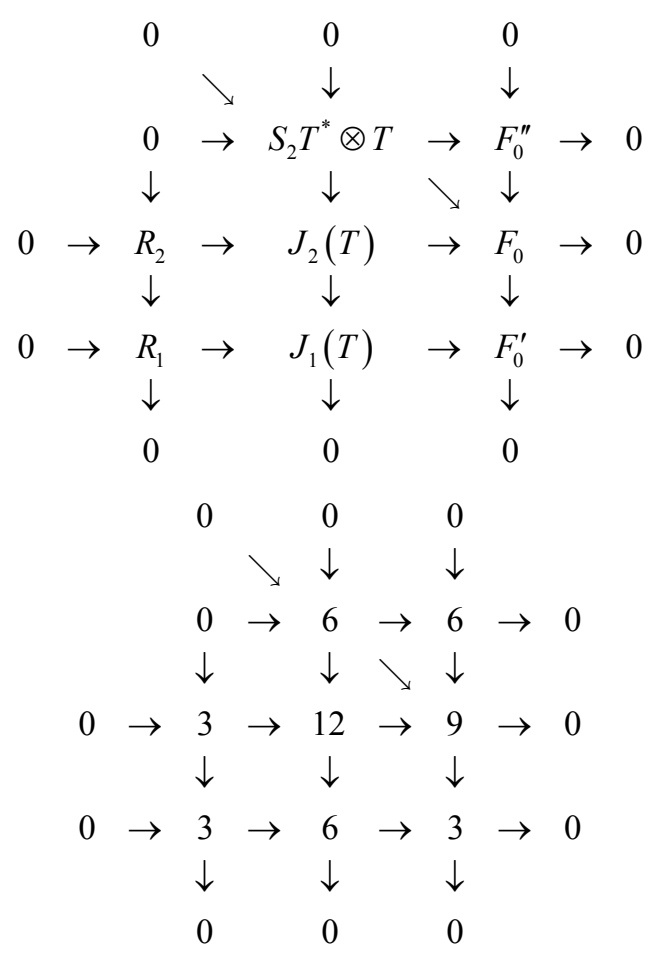

In the present situation we notice that 
$R_{2}=\rho_{1}\left(R_{1}\right)=J_{1}\left(R_{1}\right) \cap J_{2}(T) \subset J_{1}\left(J_{1}(T)\right)$ and thus $F_{0} \simeq J_{1}\left(F_{0}^{\prime}\right)$ with $F_{0}^{\prime \prime} \simeq T^{*} \otimes F_{0}^{\prime} \simeq S_{2} T^{*} \otimes T$ by counting the dimensions because we have surely $F_{0} \subset J_{1}\left(F_{0}^{\prime}\right)$ with $g_{2}=0$.

$$
\begin{aligned}
& \begin{array}{lll}
0 & 0 & 0 \\
\downarrow & \downarrow & \downarrow
\end{array} \\
& 0 \rightarrow S_{3} T^{*} \otimes T \rightarrow T^{*} \otimes F_{0} \rightarrow F_{1} \rightarrow 0 \\
& 0 \rightarrow R_{3} \rightarrow J_{3}(T) \rightarrow J_{1}\left(F_{0}\right) \rightarrow F_{1} \rightarrow 0 \\
& 0 \rightarrow R_{2} \rightarrow J_{2}(T) \rightarrow F_{0} \rightarrow 0 \\
& 0 \quad 0 \quad 0
\end{aligned}
$$

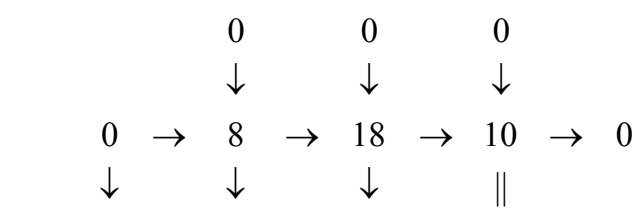

$$
\begin{aligned}
& 0 \rightarrow 3 \rightarrow 20 \rightarrow 27 \rightarrow 10 \rightarrow 0
\end{aligned}
$$

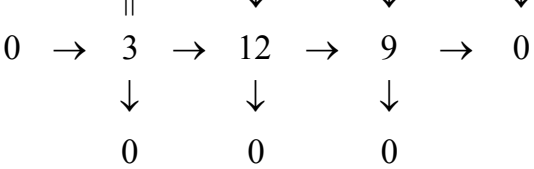

SPENCER

$$
\begin{aligned}
& \begin{array}{lll}
0 & 0 & 0 \\
\downarrow & \downarrow & \downarrow
\end{array} \\
& 0 \rightarrow \Theta \stackrel{j_{q}}{\rightarrow} \quad \begin{array}{ccccccc}
C_{0} & \stackrel{D_{1}}{\rightarrow} & C_{1} & \stackrel{D_{2}}{\rightarrow} & C_{2} & \rightarrow 0 \\
& \downarrow & & \downarrow & & \downarrow &
\end{array}
\end{aligned}
$$

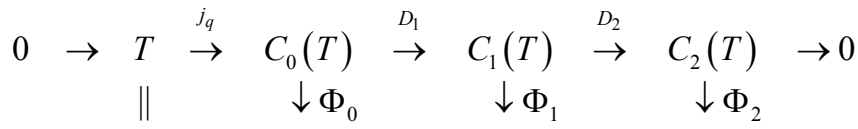

$$
\begin{aligned}
& 0 \rightarrow \Theta \rightarrow T \stackrel{\mathcal{D}}{\rightarrow} \quad \begin{array}{ccccccc}
F_{0} & \stackrel{\mathcal{D}_{1}}{\rightarrow} & F_{1} & \stackrel{\mathcal{D}_{2}}{\rightarrow} & F_{2} & \rightarrow 0 \\
& \downarrow & & \downarrow & & \downarrow & \\
& 0 & & 0 & & 0 &
\end{array}
\end{aligned}
$$

JANET

$$
\begin{aligned}
& 0 \rightarrow \tilde{\Theta} \stackrel{j_{2}}{\rightarrow} \quad 4 \quad \stackrel{D_{1}}{\rightarrow} \quad 8 \quad \stackrel{D_{2}}{\rightarrow} \quad 4 \quad \rightarrow 0 \\
& 0 \rightarrow \Theta \stackrel{j_{2}}{\rightarrow} \quad \begin{array}{llllllll}
D_{1} & \rightarrow & 6 & \stackrel{D_{2}}{\rightarrow} & 3 & \rightarrow 0 & \text { SPENCER } \\
& \downarrow & & \downarrow & & \downarrow & &
\end{array} \\
& \begin{array}{lllllllll}
2 & \stackrel{j_{2}}{\rightarrow} & 12 & \stackrel{D_{1}}{\rightarrow} & 16 & \stackrel{D_{2}}{\rightarrow} & 6 & \rightarrow 0 & \text { HYBRID } \\
& & \downarrow \Phi_{0} & & \downarrow \Phi_{1} & & \downarrow \Phi_{2} & &
\end{array} \\
& 0 \rightarrow \Theta \rightarrow 2 \stackrel{\mathcal{D}}{\rightarrow} \quad 9 \quad \stackrel{\mathcal{D}_{1}}{\rightarrow} \quad 10 \stackrel{\mathcal{D}_{2}}{\rightarrow} \quad 3 \quad \begin{array}{lllll}
D_{2} & & & &
\end{array} \\
& 0 \rightarrow \tilde{\Theta} \rightarrow 2 \stackrel{\mathcal{D}}{\rightarrow} \quad 8 \quad \stackrel{\mathcal{D}_{1}}{\rightarrow} \quad 8 \quad \stackrel{\mathcal{D}_{2}}{\rightarrow} \quad 2 \quad 2 \quad \rightarrow 0
\end{aligned}
$$




$$
\begin{aligned}
& 0 \quad 0
\end{aligned}
$$

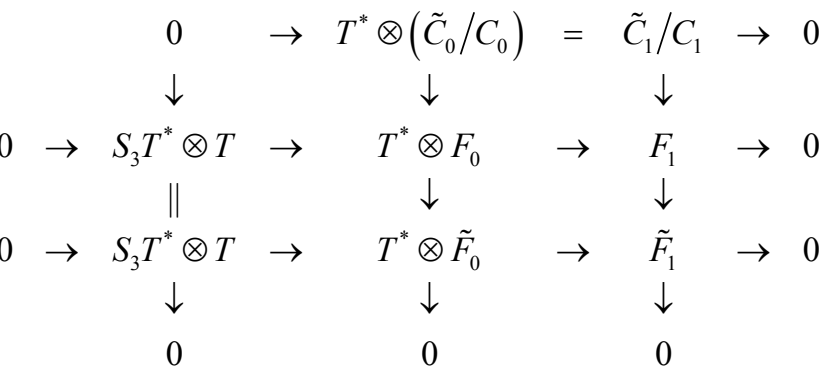

$$
\begin{aligned}
& \begin{array}{llll} 
& 0 & & 0 \\
& \downarrow & & \\
& \downarrow \\
0 \rightarrow & 2 \\
\downarrow & \downarrow & \\
& & \\
& \downarrow & &
\end{array} \\
& 0 \rightarrow 8 \rightarrow 18 \rightarrow 10 \rightarrow 0 \\
& \begin{array}{l}
\| \rightarrow \quad \downarrow \\
0 \rightarrow 16 \rightarrow 8 \\
\downarrow
\end{array} \\
& 0 \quad 0 \quad 0 \\
& \tilde{C}_{r} / C_{r} \simeq \operatorname{ker}\left(F_{r} \rightarrow \tilde{F}_{r}\right) \simeq \wedge^{r} T^{*} \otimes\left(\tilde{C}_{0} / C_{0}\right) \\
& \Rightarrow \operatorname{dim}\left(\tilde{C}_{r}\right)-\operatorname{dim}\left(C_{r}\right)=\operatorname{dim}\left(F_{r}\right)-\operatorname{dim}\left(\tilde{F}_{r}\right) \\
& \Rightarrow \operatorname{dim}\left(C_{r}\right)+\operatorname{dim}\left(F_{r}\right)=\operatorname{dim}\left(\tilde{C}_{r}\right)+\operatorname{dim}\left(\tilde{F}_{r}\right)
\end{aligned}
$$

In this new situation, we now notice that

$$
\tilde{R}_{2} \subsetneq \rho_{1}\left(\tilde{R}_{1}\right)=J_{1}\left(\tilde{R}_{1}\right) \cap J_{2}(T) \subset J_{1}\left(J_{1}(T)\right)
$$

and the induced morphism $\tilde{F}_{0} \rightarrow J_{1}\left(\tilde{F}_{0}^{\prime}\right)$ is thus no longer a monomorphism though we still have an isomorphism $\tilde{F}_{0}^{\prime \prime} \simeq S_{2} T^{*} \otimes T$ because $\tilde{g}_{2}=0$ again. Finally, we may extend such a procedure to the conformal group of space-time by considering the system of infinitesimal conformal transformations of the Minkowski metric defined by the first order system $\hat{R}_{1} \subset J_{1}(T)$ in such a way that we have the strict inclusions $R_{2} \subset \tilde{R}_{2} \subset \hat{R}_{2} \subset J_{2}(T)$ with $\operatorname{dim}\left(\hat{g}_{2}\right)=n=4$. For this, we just need to introduce the metric density $\hat{\omega}=\omega(|\operatorname{det}(\omega)|)^{-\frac{1}{n}}$ and consider the system $L(\xi) \hat{\omega}=0 \quad$ ([13]):

$$
\hat{\Omega}_{i j} \equiv \hat{\omega}_{r j} \partial_{i} \xi^{r}+\hat{\omega}_{i r} \partial_{j} \xi^{r}-\frac{2}{n} \hat{\omega}_{i j} \partial_{r} \xi^{r}+\xi^{r} \partial_{r} \hat{\omega}_{i j}=0
$$

\section{A) Control Theory:}

EXAMPLE 4.A.1: (OD control theory) In classical control theory we have $n=1$ and the only independent variable is the time, simply denoted by $x$ but we may choose any ground differential field like $K=\mathbb{Q}(x)$. In that case, we shall refer to ([5] or [14]) for the proof of the following technical results that will be used in this case (compare to [43]). Instead of the standard "upper dot" notation for derivative we shall identify the formal and the jet notations, setting thus 
$d_{x} y=d y=y_{x}$. With $m=2$, let us consider the elementary Single Input/Single Output (SISO) second order system $y_{x x}^{1}-y_{x}^{2}+a(x) y^{2}=0$ with a variable coefficient $a \in K$. The corresponding formally surjective operator is $\partial_{x x} \eta^{1}-\partial_{x} \eta^{2}+a(x) \eta^{2}=\zeta$. Treating such a system by using classical methods is not so easy when $a$ is not constant as it cannot be possible to transform it to the standard Kalman form. On the contrary, multiplying by a test function (or Lagrange multiplier) $\lambda$ and integrating by parts, we obtain the adjoint system/operator:

$$
\left\{\begin{array}{l}
y^{1} \rightarrow \lambda_{x x}=\mu^{1} \\
y^{2} \rightarrow \lambda_{x}+a \lambda=\mu^{2}
\end{array}\right.
$$

This system has a trivially involutive zero symbol but is not even formally integrable and we have to consider:

$$
\begin{cases}\lambda_{x x}=\mu^{1} & 1 \\ \lambda_{x}+a \lambda=\mu^{2} & \bullet \\ \left(\partial_{x} a-a^{2}\right) \lambda=\mu_{x}^{2}-\mu^{1}-a \mu^{2} & \bullet\end{cases}
$$

We have thus two possibilities:

- We have $a_{x}-a^{2} \neq 0$ and the adjoint system has the only zero solution, that is the adjoint operator is injective. In this case $N=0$ and thus $t(M)=\operatorname{ext}^{1}(N)=0$ that is $M$ is torsion-free. However, as $n=1$ it follows that $D=K[d]$ is a principal ideal ring which is therefore free and thus projective ([26] [30]), that is $M$ is torsion-free if and only if $N=0$ and the system is controllable.

- The Riccati equation $a_{x}-a^{2}=0$ is satisfied, for example if $a=-1 / x$, and we get the CC $\mu_{x}^{2}-\mu^{1}-a \mu^{2}=0$. Multiplying by a test function $\xi$ and integrating by parts, we get the adjoint operator:

$$
\left\{\begin{array}{l}
\mu^{1} \rightarrow-\xi=\eta^{1} \\
\mu^{2} \rightarrow-\xi_{x}-a \xi=\eta^{2}
\end{array}\right.
$$

with only one first order generating CC, namely $\partial_{x} \eta^{1}-\eta^{2}+a \eta^{1}=0$. It follows that $N \neq 0 \Rightarrow \operatorname{ext}^{1}(N) \neq 0$ is a torsion module generated by the residue of $z=y_{x}^{1}-y^{2}+a y^{1}$, even though $y^{1}$ and $y^{2}$ are free separately and $M$ is not pure. We obtain indeed a torsion element as we can check at once that $z_{x}-a z=0$ and wish good luck for control people to recover simply this result even on such an elementary example because the Kalman criterion is only working for systems with constant coefficients (compare [5] and [43]).

EXAMPLE 4.A.2: (PD control theory) With $n=2$, let us consider the (trivially involutive) inhomogeneous single first order PD equations with two independent variables $\left(x^{1}, x^{2}\right)$, two unknown functions $\left(\eta^{1}, \eta^{2}\right)$ and a second member $\zeta$ :

$$
\partial_{2} \eta^{1}-\partial_{1} \eta^{2}+x^{2} \eta^{2}=\zeta \Leftrightarrow \mathcal{D}_{1} \eta=\zeta
$$

The ring of differential operators is $D=K\left[d_{1}, d_{2}\right]$ with $K=\mathbb{Q}\left(x^{1}, x^{2}\right)$. Mul- 
tiplying on the left by a test function $\lambda$ and integrating by parts, the corresponding adjoint operator is described by:

$$
\left\{\begin{array}{l}
\eta^{1} \rightarrow-\partial_{2} \lambda=\mu^{1} \\
\eta^{2} \rightarrow \partial_{1} \lambda+x^{2} \lambda=\mu^{2}
\end{array} \Leftrightarrow \quad \operatorname{ad}\left(\mathcal{D}_{1}\right) \lambda=\mu\right.
$$

Using crossed derivatives, this operator is injective because $\lambda=\partial_{2} \mu^{2}+\partial_{1} \mu^{1}+x^{2} \mu^{1}$ and we even obtain a lift $\lambda \rightarrow \mu \rightarrow \lambda$. Substituting, we get the two CC:

$$
\left\{\begin{array}{l}
\partial_{22} \mu^{2}+\partial_{12} \mu^{1}+x^{2} \partial_{2} \mu^{1}+2 \mu^{1}=v^{1} \\
\partial_{12} \mu^{2}+\partial_{11} \mu^{1}+2 x^{2} \partial_{1} \mu^{1}+x^{2} \partial_{2} \mu^{2}+\left(x^{2}\right)^{2} \mu^{1}-\mu^{2}=v^{2}
\end{array}\right.
$$

This system is involutive and the corresponding generating CC for the second member $\left(v^{1}, v^{2}\right)$ is:

$$
\partial_{2} v^{2}-\partial_{1} v^{1}-x^{2} v^{1}=0
$$

Therefore $v^{2}$ is differentially dependent on $v^{1}$ but $v^{1}$ is also differentially dependent on $v^{2}$. Multiplying on the left by a test function $\theta$ and integrating by parts, the corresponding adjoint system of PD equations is:

$$
\left\{\begin{array}{l}
v^{1} \rightarrow \partial_{1} \theta-x^{2} \theta=\xi^{1} \\
v^{2} \rightarrow-\partial_{2} \theta=\xi^{2}
\end{array} \Leftrightarrow \quad a d\left(\mathcal{D}_{-1}\right) \theta=\xi\right.
$$

Multiplying now the first equation by the test function $\xi^{1}$, the second equation by the test function $\xi^{2}$, adding and integrating by parts, we get the canonical parametrization $D \xi=\eta$ :

$$
\left\{\begin{array}{l}
\mu^{2} \rightarrow \partial_{22} \xi^{1}+\partial_{12} \xi^{2}-x^{2} \partial_{2} \xi^{2}-2 \xi^{2}=\eta^{2} \\
\mu^{1} \rightarrow \partial_{12} \xi^{1}-x^{2} \partial_{2} \xi^{1}+\xi^{1}+\partial_{11} \xi^{2}-2 x^{2} \partial_{1} \xi^{2}+\left(x^{2}\right)^{2} \xi^{2}=\eta^{1}
\end{array}\right.
$$

of the initial system with zero second member. This system is involutive and the kernel of this parametrization has differential rank equal to 1 because $\xi^{1}$ or $\xi^{2}$ can be given arbitrarily.

Keeping now $\xi^{1}=\xi$ while setting $\xi^{2}=0$, we get the first second order minimal parametrization $\xi \rightarrow\left(\eta^{1}, \eta^{2}\right)$ :

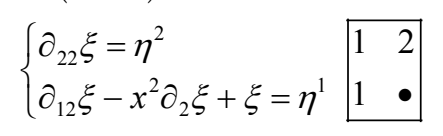

This system is again involutive and the parametrization is minimal because the kernel of this parametrization has differential rank equal to 0 . With a similar comment, setting now $\xi^{1}=0$ while keeping $\xi^{2}=\xi^{\prime}$, we get the second order minimal parametrization $\xi^{\prime} \rightarrow\left(\eta^{1}, \eta^{2}\right)$ :

$$
\left\{\begin{array}{l}
\partial_{11} \xi^{\prime}-2 x^{2} \partial_{1} \xi^{\prime}+\left(x^{2}\right)^{2} \xi^{\prime}=\eta^{1} \\
\partial_{12} \xi^{\prime}-x^{2} \partial_{2} \xi^{\prime}-2 \xi^{\prime}=\eta^{2}
\end{array}\right.
$$

which is again easily seen to be involutive by exchanging $x^{1}$ with $x^{2}$.

With again a similar comment, setting now $\xi^{1}=\partial_{1} \phi, \xi_{2}=-\partial_{2} \phi$ in the ca- 
nonical parametrization, we obtain the third different second order minimal parametrization:

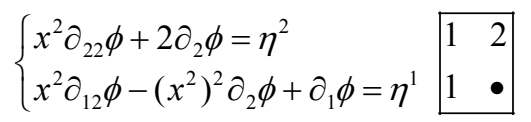

We are now ready for understanding the meaning and usefulness of what we have called "relative parametrization" in ([4]) by imposing the differential constraint $\partial_{2} \xi^{1}+\partial_{1} \xi^{2}=0$ which is compatible as we obtain indeed the new first order relative parametrization:

$$
\left\{\begin{array}{l}
\partial_{2} \xi^{1}+\partial_{1} \xi^{2}=0 \\
-x^{2} \partial_{2} \xi^{2}-2 \xi^{2}=\eta^{2} \\
-x^{2} \partial_{1} \xi^{2}+\left(x^{2}\right)^{2} \xi^{2}+\xi^{1}=\eta^{1}
\end{array}\right.
$$

with 2 equations of class 2 (thus with class 2 full) and only 1 equation of class 1 .

In a different way, we may add the differential constraint $\partial_{1} \xi^{1}+\partial_{2} \xi^{2}=0$ but we have to check that it is compatible with the previous parametrization. For this, we have to consider the following second order system which is easily seen to be involutive with 2 second order equations of (full) class 2, (only) 2 second order equations of class 1 and 1 equation of order 1 :

$$
\left\{\begin{array}{l}
\partial_{22} \xi^{2}+\partial_{12} \xi^{1}=0 \\
\partial_{22} \xi^{1}+\partial_{12} \xi^{2}-x^{2} \partial_{2} \xi^{2}-2 \xi^{2}=\eta^{2} \\
\partial_{12} \xi^{2}+\partial_{11} \xi^{1}=0 \\
\partial_{12} \xi^{1}-x^{2} \partial_{2} \xi^{1}+\xi^{1}+\partial_{11} \xi^{2}-2 x^{2} \partial_{1} \xi^{2}+\left(x^{2}\right)^{2} \xi^{2}=\eta^{1} \\
\partial_{2} \xi^{2}+\partial_{1} \xi^{1}=0
\end{array} \mid \begin{array}{ll}
1 & 2 \\
1 & 2 \\
1 & \bullet \\
1 & \bullet \\
\bullet & \bullet
\end{array}\right.
$$

The 4 generating CC only produce the desired system for $\left(\eta^{1}, \eta^{2}\right)$ as we wished.

We cannot impose the condition $\mathcal{D}_{-1} \theta=\xi$ already found as it should give the identity $0=\eta$.

It is however also important to notice that the strictly exact long exact sequence:

$$
0 \rightarrow D \stackrel{\mathcal{D}_{1}}{\longrightarrow} D^{2} \stackrel{\mathcal{D}}{\longrightarrow} D^{2} \stackrel{\mathcal{D}_{-1}}{\longrightarrow} D \rightarrow 0
$$

splits because we have a lift $\zeta \rightarrow \eta \rightarrow \zeta$, namely:

$$
\zeta \rightarrow\left(-\partial_{1} \zeta+x^{2} \zeta=\eta^{1},-\partial_{2} \eta^{2}=\eta^{2}\right) \rightarrow \partial_{2} \eta^{1}-\partial_{1} \eta^{2}+x^{2} \eta^{2}=\zeta
$$

We have thus an isomorphism $D^{2} \simeq D \oplus M$ in the resolution $0 \rightarrow D \stackrel{\mathcal{D}_{1}}{\longrightarrow} D^{2} \stackrel{p}{\longrightarrow} M \rightarrow 0$ and all the differential modules defined from the operators involved are projective, thus torsion-free or 0 -pure with vanishing $r$-extension modules $\operatorname{ext}^{r}()=0, \forall r \geq 1$.

As an exercise, we finally invite the reader to study the situation met with the system $\partial_{2} \eta^{1}-\partial_{1} \eta^{2}+a(x) \eta^{2}$ whenever $a \in K$ (Hint. The controllability condition is now $\left.\partial_{1} a \neq 0\right)$. The comparison with the previous OD case needs no comment. 


\section{B) Electromagnetism:}

Most physicists know the Maxwell equations in vacuum, eventually in dielectrics and magnets, but are largely unaware of the more delicate constitutive laws involved in field-matter couplings like piezzoelectricity, photoelasticity or streaming birefringence. In particular they do not know that the phenomenological laws of these phenomena have been given ... by Maxwell ([7]). The situation is even more critical when they deal with invariance properties of Maxwell equations because of the previous comments ([44]). Therefore, we shall first quickly recall what the use of adjoint operators and differential duality can bring when studying Maxwell equations as a first step before providing comments on the so-called gauge condition brought by the Danish physicist Ludwig Lorenz in 1867 and not by Hendrik Lorentz with name associated with the Lorentz transformations.

Though it is quite useful in actual practice, the following approach to Maxwell equations cannot be found in any textbook. Namely, avoiding any variational calculus based on given Minkowski constitutive laws $\mathcal{F} \sim F$ between field $F$ and induction $\mathcal{F}$ for dielectric or magnets, let us use differential duality and define the first set $M_{1}$ of Maxwell equations by $d: \wedge^{2} T^{*} \rightarrow \wedge^{3} T^{*}$ while the second set $M_{2}$ will be defined by $a d(d): \wedge^{4} T^{*} \otimes \wedge^{2} T \rightarrow \wedge^{4} T^{*} \otimes T$ with $d: T^{*} \rightarrow \wedge^{2} T^{*}$, in a totally independent and intrinsic manner, using now contravariant tensor densities in place of covariant tensors. As we have already proved since a long time in ([3] [7] [13] [14] [33] [40]), the key result is that these two sets of Maxwell equations are invariant by any diffeomorphism, contrary to what is generally believed ([44]). We recapitulate below this procedure in the form of a (locally exact) differential sequence and its (locally exact) formal adjoint sequences where the dotted down arrow in the left square is the standard composition of operators:

$$
\begin{array}{ccccccc}
\text { potential } & \stackrel{d}{\rightarrow} & \text { field } & \rightarrow & \text { induction } & \stackrel{\operatorname{ad}(d)}{ } & \text { current } \\
A & \rightarrow & F & \rightarrow & \mathcal{F} & \rightarrow & \mathcal{J}
\end{array}
$$

which is responsible for EM waves, though it is equivalent to the composition in the right square:

$$
\text { pseudopotential } \stackrel{a d(d)}{\rightarrow} \text { induction } \rightarrow \text { field } \stackrel{d}{\rightarrow} \wedge^{3} T^{*}
$$

The main difference is that we need to set $\mathcal{J}=0$ in the first approach because of $M_{2}$ while we get automatically such a vanishing assumption in the second approach because of $M_{1}$, avoiding therefore the Lorenz condition as in ([35], Remark 5.5) and below.

$$
\begin{gathered}
\text { potential }=\left(A_{i}\right) \quad \stackrel{d}{\rightarrow}\left(\partial_{i} A_{j}-\partial_{j} A_{i}=F_{i j}\right)=\text { field } \stackrel{M_{1}}{\rightarrow}\left(\partial_{i} F_{j k}+\partial_{j} F_{k i}+\partial_{k} F_{i j}=0\right) \\
\text { current }=\left(\partial_{i} \mathcal{F}^{i j}=\mathcal{J}^{j}\right) \stackrel{M_{2}}{\leftarrow} \quad\left(\mathcal{F}^{i j}\right)=\text { induction } \stackrel{\operatorname{ad}(d)}{\leftarrow} \quad \text { pseudopotential }
\end{gathered}
$$




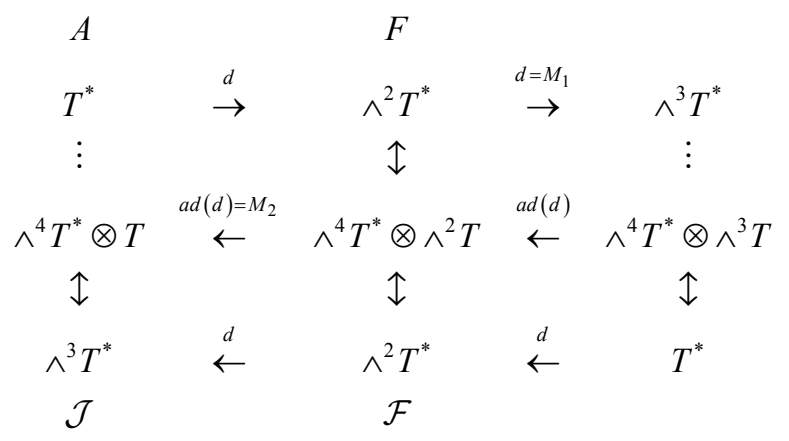

Using symbolic notations with an Euclidean metric instead of the Minkowski one because they are both locally constant while using the constitutive law $\mathcal{F}=F$ for simplicity in vacuum while raising or lowering the indices by means of the metric, we have the parametrization $d_{i} A_{j}-d_{j} A_{i}=F_{i j}$ and obtain by composition in the left upper square:

$$
\begin{aligned}
& d_{i}\left(d^{i} A^{j}-d^{j} A^{i}\right)=d_{i} d^{i} A^{j}-d^{j}\left(d_{i} A^{i}\right)=\mathcal{J}^{j} \\
& \Rightarrow d_{j}\left(d_{i} d^{i} A^{j}-d^{j} d_{i} A^{i}\right)=d_{j} \mathcal{J}^{j}=0
\end{aligned}
$$

with implicit summations on $i$ and $j$. However, such a result does not prove at all that the density of current does not satisfy other CC. Nevertheless, we have:

LEMMA 4.B.1: The system $d_{i} d^{i} A^{j}-d^{j}\left(d_{i} A^{i}\right)=\mathcal{J}^{j}$ is involutive whenever $d_{j} \mathcal{J}^{j}=0$ but the differential module defined by the corresponding homogeneous system is not torsion-free.

Proof: When $\mathcal{J}=0$, this second order system is formally integrable because it is homogeneous. However, even if we know a priori that necessarily $d_{j} \mathcal{J}^{j}=0$, it is not evident that such a condition is also sufficient, contrary to what is claimed in the literature. When $n=4$, using the Euclidean metric for simplicity, one can rewrite the system in the form:

$\left\{\begin{array}{l}d_{44} A^{3}+d_{33} A^{3}+d_{22} A^{3}+d_{11} A^{3}-d_{3}\left(d_{4} A^{4}+d_{3} A^{3}+d_{2} A^{2}+d_{1} A^{1}\right)=\mathcal{J}^{3} \\ d_{44} A^{2}+d_{33} A^{2}+d_{22} A^{2}+d_{11} A^{3}-d_{2}\left(d_{4} A^{4}+d_{3} A^{3}+d_{2} A^{2}+d_{1} A^{1}\right)=\mathcal{J}^{2} \\ d_{44} A^{1}+d_{33} A^{1}+d_{22} A^{1}+d_{11} A^{3}-d_{1}\left(d_{4} A^{4}+d_{3} A^{3}+d_{2} A^{2}+d_{1} A^{1}\right)=\mathcal{J}^{1} \\ d_{34} A^{3}+d_{24} A^{2}+d_{14} A^{1}-d_{33} A^{4}-d_{22} A^{4}-d_{11} A^{4}=-\mathcal{J}^{4}\end{array}\right.$

Let us check that the second order symbol is involutive with three equations of class 4 and only one equation of class 3. Indeed, we have successively:

$$
\begin{aligned}
& d_{344} A^{3}+d_{244} A^{2}+d_{144} A^{1}-d_{334} A^{4}-d_{224} A^{4}-d_{114} A^{4}=-d_{4} \mathcal{J}^{4} \\
& -d_{344} A^{3}-d_{333} A^{3}-d_{223} A^{3}-d_{113} A^{3}+d_{334} A^{4}+d_{333} A^{3}+d_{233} A^{2}+d_{133} A^{1}=-d_{3} \mathcal{J}^{3} \\
& -d_{244} A^{2}-d_{233} A^{2}-d_{222} A^{2}-d_{112} A^{2}+d_{224} A^{4}+d_{223} A^{3}+d_{222} A^{2}+d_{122} A^{1}=-d_{2} \mathcal{J}^{2} \\
& -d_{144} A^{1}-d_{133} A^{1}-d_{122} A^{1}-d_{111} A^{1}+d_{114} A^{4}+d_{113} A^{3}+d_{112} A^{2}+d_{111} A^{1}=-d_{1} \mathcal{J}^{1}
\end{aligned}
$$

Summing the four equations, the left member is vanishing and we get the desired only CC. The four characters are $(1<7<12<16)$ and we check that $\operatorname{dim}\left(g_{2}\right)=1+7+12+16=36=(10 \times 4)-4$. This procedure cannot be avoided though it cannot be found in the literature.

In addition, rewriting the homogeneous system in the form $d_{i} d_{i} A_{j}-d_{i} d_{j} A_{i}=0$ 
with implicit summation on $i$, we obtain successively ([10]):

$$
\begin{aligned}
d_{i} d_{i} F_{j k} & =d_{i} d_{i}\left(d_{j} A_{k}-d_{k} A_{j}\right) \\
& =d_{j}\left(d_{i} d_{i} A_{k}\right)-d_{k}\left(d_{i} d_{i} A_{j}\right) \\
& =d_{j}\left(d_{i} d_{k} A_{i}\right)-d_{k}\left(d_{i} d_{j} A_{i}\right) \\
& =d_{j} d_{k}\left(d_{i} A_{i}\right)-d_{k} d_{j}\left(d_{i} A_{i}\right) \\
& =0
\end{aligned}
$$

It follows that each component of the field is a torsion element of the corresponding differential module $M$, which is killed by the Dalembertian. Accordingly, $M$ is not torsion-free and thus not pure because we have just proved that $r k_{D}(M)=1$. In particular, each component of the potential is free by itself. Such a situation is similar to the one met in the study of Single Input/Single Output (SISO) ordinary differential control systems. For example, if we have $y_{x x}-u_{x}=0$, then $z=y_{x}-u$ is a torsion element with $z_{x}=0$ even though both the input $u$ and the output $y$ are free separately. Bringing the system to first order by choosing the new unknowns $\left(z^{1}=y, z^{2}=y_{x}, z^{3}=u\right)$, we obtain the Spencer form over $K=\mathbb{Q}$ :

$$
z_{x}^{1}-z^{2}=0, z_{x}^{2}-z_{x}^{3}=0
$$

Setting a new $\bar{z}^{1}=z^{1}, \bar{z}^{2}=z^{2}-z^{3}, \bar{z}^{3}=z^{3}$, we obtain the reduced Spencer form:

$$
\bar{z}_{x}^{1}-\bar{z}^{2}-\bar{z}^{3}=0, \bar{z}_{x}^{2}=0 \Leftrightarrow \bar{z}=A z+B u \Rightarrow r k_{K}(B, A B)=1<2
$$

which is a Kalman form because no jet of the input $\bar{z}^{3}=z^{3}=u$ is indeed appearing.

Finally, the character $\alpha_{2}^{n}$ is obtained by considering $d_{n n} A_{j}-d_{j n} A_{n}$ for the equation giving $\mathcal{J}_{j}$. For $\mathcal{J}_{n}$ we get $d_{n n} A_{n}-d_{n n} A_{n}=0$ and thus $\alpha_{2}^{n}=n-(n-1)=1$ a result showing that the corresponding differential module has rank 1 and there is thus only one CC, namely $d_{j} \mathcal{J}^{j}=0$ with implicit summation on $j$.

Q.E.D.

We now prove that we may add the Lorenz condition $d_{i} A^{i}=0$ to bring the rank to zero. Indeed, we have now the inhomogeneous system $d_{i} d^{i} A^{j}=\mathcal{J}^{j}$ and the differential constraint thus brought is compatible with the conservation of current. The corresponding homogeneous system obtained by adding the Lorenz constraint has second order symbol obtained by considering both $d_{i} d^{i} A^{j}=0$ and $d_{i j} A^{i}=0$ or $d_{i j} A^{j}=0$. We obtain therefore $d_{n n} A_{j}=0, d_{n n} A_{n}=0$ showing that we have now $\alpha_{2}^{n}=0$ and a torsion differential module. As a more important and effective result that does not seem to be known, we have:

PROPOSITION 4.B.2: When $n=4$, the system:

$$
\left\{\begin{array}{l}
\Psi^{j} \equiv d_{44} A^{j}+\cdots+d_{11} A^{j}=\mathcal{J}^{j} \\
d_{4} \Phi-\Psi^{4} \equiv d_{34} A^{3}+d_{24} A^{2}+d_{14} A^{1}-d_{33} A^{4}-d_{22} A^{2}-d_{11} A^{4}=-\mathcal{J}^{4} \\
d_{3} \Phi \equiv d_{34} A^{4}+\cdots+d_{13} A^{1}=0 \\
d_{2} \Phi \equiv d_{24} A^{4}+\cdots+d_{12} A^{1}=0 \\
d_{1} \Phi \equiv d_{14} A^{4}+\cdots+d_{11} A^{1}=0 \\
\Phi \equiv d_{4} A^{4}+\cdots+d_{1} A^{1}=0
\end{array} \mid \begin{array}{llll}
1 & 2 & 3 & 4 \\
1 & 2 & 3 & \bullet \\
1 & 2 & 3 & \bullet \\
1 & 2 & \bullet & \bullet \\
1 & \bullet & \bullet & \bullet \\
\bullet & \bullet & \bullet & \bullet
\end{array}\right.
$$


is involutive with four equations of class 4 , two equations of class 3 , one equation of class 2 and one equation of class 1 . The 11 resulting CC only provide the conservation of current.

Proof. Using the corresponding Janet tabular on the right, one can check at once that the $4 \mathrm{CC}$ brought by the only first order equation $\Phi=0$ do not bring anything new, as they amount to crossed derivatives, and that we are only left with the 4 upper dots on the right side. However, for $i=1,2,3$, we have $d_{4}\left(d_{i} \Phi\right)=d_{i}\left(d_{4} \Phi-\Psi^{4}\right)+d_{i} \Psi^{4}$ and we are thus only left with a single CC, getting successively:

$$
\begin{gathered}
d_{4}\left(d_{4} \Phi-\Psi^{4}\right) \equiv d_{344} A^{3}+d_{244} A^{2}+d_{144} A^{1}-d_{334} A^{4}-d_{224} A^{2}-d_{114} A^{1} \\
-d_{3} \Psi^{3} \equiv-d_{344} A^{3}-d_{333} A^{3}-d_{223} A^{3}-d_{113} A^{3} \\
-d_{2} \Psi^{2} \equiv-d_{244} A^{2}-d_{233} A^{2}-d_{222} A^{2}-d_{112} A^{2} \\
-d_{1} \Psi^{1} \equiv-d_{144} A^{1}-d_{133} A^{1}-d_{122} A^{1}-d_{111} A^{1} \\
d_{3}\left(d_{3} \Phi\right) \equiv d_{334} A^{4}+d_{333} A^{3}+d_{233} A^{2}+d_{133} A^{1} \\
d_{2}\left(d_{2} \Phi\right) \equiv d_{224} A^{4}+d_{223} A^{3}+d_{222} A^{2}+d_{122} A^{1} \\
d_{1}\left(d_{1} \Phi\right) \equiv d_{114} A^{4}+d_{113} A^{3}+d_{112} A^{2}+d_{111} A^{1}
\end{gathered}
$$

Summing these 7 equations, we are left with the identity $-\left(d_{44} \Phi+\cdots+d_{11} \Phi\right)+d_{j} \Psi^{j}=d_{j} \mathcal{J}^{j}=0$. It is important to notice that no other procedure can prove that we have an involutive symbol in $\delta$-regular coordinates and this is the only way to compute effectively all the four characters $(0<6<11<15)$ with $6+11+15=32=(4 \times 10)-(4+4)$ for the dimension of the symbol of order 2, a result not evident at first sight. Accordingly, the so-called Lorenz gauge condition is only a pure "artifact" amounting to a relative minimum parametrization with no important physical meaning as it can be avoided by using only the EM field $F$ ([6] [10] [45]).

Q.E.D.

REMARK 4.B.3: Using the exactness of the Euler-Poincare characteristic for the Poincaré sequence and its adjoint sequence, we have:

$$
\begin{gathered}
0=1-n+n(n-1) / 2-r k_{D}\left(d=M_{1}\right) \Rightarrow r k_{D}\left(d=M_{1}\right)=(n-1)(n-2) / 2 \\
0=1-n+n(n-1) / 2-r k_{D}\left(d=M_{2}\right) \Rightarrow r k_{D}\left(a d(d)=M_{2}\right)=(n-1)(n-2) / 2
\end{gathered}
$$

by counting the ranks from left to right in the upper row and then from right to left in lower row of the previous diagram. We obtain therefore:

$$
\begin{aligned}
& r k_{D}(d)+r k_{D}\left(d=M_{1}\right) \\
& =r k_{D}\left(\operatorname{ad}(d)=M_{2}\right)+r k_{D}(\operatorname{ad}(d))=3+3=\operatorname{dim}\left(\wedge^{2} T^{*}\right)=6
\end{aligned}
$$

when $n=4$ by using successively the exactness of the upper and lower rows of the diagram. Hence, the image of the dotted arrow on the left is equal to the image of $a d(d)=M_{2}$, a result explaining why both operators have the same CC, namely the conservation of current obtained by extending the previous diagram one step more to the left. As the linear Spencer sequence for a Lie group action (isometries, isometries plus dilatation, conformal isometries in Example 4.1) is 
(locally) isomorphic to the tensor product of the Poincaré sequence by a finite dimensional Lie algebra $(10<11<15$ when $n=4$ in Example 4.1), the same comment remains valid. This result justifies by itself the specific importance of the linear Spencer sequence for infinitesimal Lie equations.

Such a new approach to a classical result is nevertheless bringing a totally unsatisfactory consequence. Using the well known correspondence between electromagnetism (EM) and elasticity (EL) used for all engineering computations with finite elements:

$$
\begin{aligned}
& \text { EM potential } \leftrightarrow \text { EL displacement, } \quad \text { EM field } \leftrightarrow \text { EL strain, } \\
& \text { EM induction } \leftrightarrow \text { EL stress }
\end{aligned}
$$

where EL means elasticity, and instead of the left upper square in the diagram, ... we have to consider the right upper square.

We finally prove that the use of the linear and nonlinear Spencer operators drastically changes the previous standard procedure in a way that could not even be imagined with classical methods. For such a purpose, we make a few comments on the implicit summation appearing in differential duality. For example, we have, up to a divergence:

$$
\mathcal{X}_{k}^{, r} X_{, r}^{k}=\mathcal{X}_{k}^{, r}\left(\partial_{r} \xi^{k}-\xi_{r}^{k}\right)=-\partial_{r}\left(\mathcal{X}_{k}^{, r}\right) \xi^{k}-\mathcal{X}_{k}^{, r} \xi_{r}^{k}+\cdots
$$

In the conformal situation, we have $\xi_{1}^{1}=\xi_{2}^{2}=\cdots=\xi_{n}^{n}=\frac{1}{n} \xi_{r}^{r}$ and obtain therefore, as factor of the firs jets:

$$
\mathcal{X}_{1}^{, 1} \xi_{1}^{1}+\mathcal{X}_{2}^{, 2} \xi_{2}^{2}+\cdots+\mathcal{X}_{n}^{, n} \xi_{n}^{n}=\left(\mathcal{X}_{r}^{, r}\right) \frac{1}{n} \xi_{r}^{r}=\left(\mathcal{X}_{r}^{, r}\right) \xi_{1}^{1}
$$

Going to the next order, we get as in ([26]), up to a divergence:

$$
\mathcal{X}_{1}^{1, r} \partial_{r} \xi_{1}^{1}=-\left(\partial_{r} \mathcal{X}_{1}^{1, r}\right) \xi_{1}^{1}+\cdots
$$

Collecting the results and changing the sign, we obtain for the first time the Cosserat equation for the dilatation, namely the so-called virial equation that we provided in 2016 ([34], p. 35):

$$
\partial_{r} \mathcal{X}_{1}^{1, r}+\mathcal{X}_{r}^{, r}=0
$$

generalizing the well known Cosserat equations for the rotations provided in 1909 ([46], p. 137):

$$
\partial_{r} \mathcal{X}^{i j, r}+\mathcal{X}^{i, j}-\mathcal{X}^{j, i}=0
$$

As for EM, substituting $\partial_{i} \xi_{r j}^{r}-\partial_{j} \xi_{r i}^{r}$ in the dual sum $\mathcal{F}^{i<j} F_{i<j}=\frac{1}{2} \mathcal{F}^{i j} F_{i j}$ and integrating by parts, we get a part of the Cosserat equations for the elations, namely:

$$
\partial_{r} \mathcal{F}^{i r}-\mathcal{J}^{i}=0 \Rightarrow \partial_{i} \mathcal{J}^{i}=0 \Rightarrow \mathcal{X}_{r}^{, r}=0
$$

saying that the trace of the EM impulsion-energy tensor must vanish ([45], p. 37).

REMARK 4.B.4: When $g_{q+1}=0$, introducing the linear Spencer sequence 
with Spencer bundles $C_{r}=\wedge^{r} T^{*} \otimes \hat{R}_{q}$, we obtain the following diagram describing the Cosserat procedure ([4] [40] [46]):

\begin{tabular}{ccccc} 
potential & \multicolumn{5}{c}{ field } & \\
$C_{0}$ & $\stackrel{D_{1}}{\rightarrow}$ & $C_{1}$ & $\stackrel{D_{2}}{\rightarrow}$ & $C_{2}$ \\
$\vdots$ & & $\uparrow$ & & $\vdots$ \\
$\wedge^{n} T^{*} \otimes C_{0}^{*}$ & $\stackrel{a d\left(D_{1}\right)}{\leftarrow}$ & $\wedge^{n} T^{*} \otimes C_{1}^{*}$ & $\stackrel{\operatorname{ad}\left(D_{2}\right)}{\leftarrow}$ & $\wedge^{n} T^{*} \otimes C_{2}^{*}$ \\
$\uparrow$ & & $\uparrow$ & & $\uparrow$ \\
$\wedge^{n} T^{*} \otimes M_{q}$ & $\stackrel{a d\left(D_{1}\right)}{\leftarrow}$ & $\wedge^{n-1} T^{*} \otimes M_{q}$ & $\stackrel{\operatorname{ad}\left(D_{2}\right)}{\leftarrow}$ & $\wedge^{n-2} T^{*} \otimes M_{q}$ \\
& & induction & & pseudopotential
\end{tabular}

where we have used the isomorphism $R_{q}^{*} \simeq M_{q}$. It just remains to consider the various Spencer bundles $C_{r} \subset \tilde{C}_{r} \subset \hat{C}_{r}$ that we have already considered with $q=2$ (see [17] for an explicit example).

We sum up all these results in the following tabular only depending on the Spencer operator:

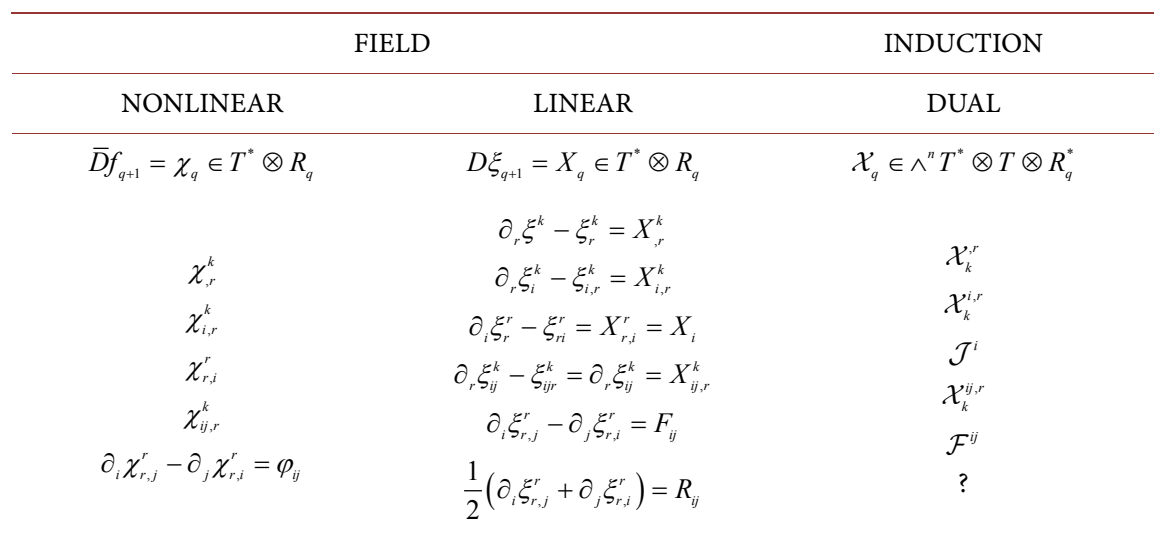

We notice that $F=\left(F_{i j}\right) \in \wedge^{2} T^{*}, R=\left(R_{i j}\right) \in S_{2} T^{*}$ and the part of the first Spencer bundle made by the 1 -forms with value in the $n=4$ elations provides the splitting:

$$
(F, R) \in \wedge^{2} T^{*} \oplus S_{2} T^{*} \simeq T^{*} \otimes T^{*}
$$

because of the well known exactness of the Spencer $\delta$-sequence:

$$
0 \rightarrow S_{2} T^{*} \stackrel{\delta}{\longrightarrow} T^{*} \otimes T^{*} \stackrel{\delta}{\longrightarrow} \wedge^{2} T^{*} \rightarrow 0
$$

As a byproduct, the surprising shift of exterior powers is simply obtained by applying the Spencer $\delta$-map to $\hat{C}_{r} / \tilde{C}_{r}=\wedge^{r} T^{*} \otimes \hat{g}_{2} \simeq \wedge^{r} T^{*} \otimes T^{*}$ because $\delta\left(\wedge^{r} T^{*} \otimes T^{*}\right) \subset \wedge^{(r+1)} T^{*}$.

\section{C) General Relativity:}

Roughly speaking, we shall say that a parametrization of an operator is $m i$ nimal if its corresponding operator defines a torsion module or, equivalently, if the kernel of the parametrizing operator has differential rank equal to 0 . It is not so well known even today that, up to an isomorphism, the Cauchy stress opera- 
tor essentially admits only one parametrization in dimension $n=2$ which is minimum but the situation is quite different in dimension $n=3$. Indeed, the parametrization found by E. Beltrami in 1892 with 6 potentials ([9]) is not minimal as the kernel of the Beltrami operator has differential rank 3 while the two other parametrizations respectively found by J.C. Maxwell in 1870 and by G. Morera in 1892 are both minimal with only 3 potentials even though they are quite different because the first is cancelling 3 among the 6 potentials while the other is cancelling the 3 others. In particular, we point out the technical fact that it is quite difficult to prove that the Morera parametrization is providing an involutive system. These three tricky examples are proving that the possibility to exhibit different parametrizations of the stress equations that we have presented has surely nothing to do with the proper mathematical background of elasticity theory as it provides an explicit application of double differential duality in differential homological algebra. Also, the example presented in Section 3. A is proving that the existence of many different minimal parametrizations has surely nothing to do with the mathematical foundations of control theory. Similarly, we have just seen in the previous section that the so-called Lorenz condition has surely nothing to do with the mathematical foundations of EM. Such a comment will be now extended in a natural manner to GR.

With standard notations, denoting by $\Omega \in S_{2} T^{*}$ a perturbation of the non-degenerate metric $\omega$, it is well known (see [8] [10] and [47] for more details) that the linearization of the Ricci tensor $R=\left(R_{i j}\right) \in S_{2} T^{*}$ over the Minkowski metric, considered as a second order operator $\Omega \rightarrow R$, may be written with four terms as:

$$
2 R_{i j}=\omega^{r s}\left(d_{i j} \Omega_{r s}+d_{r s} \Omega_{i j}-d_{r i} \Omega_{s j}-d_{s j} \Omega_{r i}\right)=2 R_{j i}
$$

Multiplying by test functions $\left(\lambda^{i j}\right) \in \wedge^{4} T^{*} \otimes S_{2} T$ and integrating by parts on space-time, we obtain the following four terms describing the so-called gravitational waves equations.

$$
\left(\square \lambda^{r s}+\omega^{r s} d_{i j} \lambda^{i j}-\omega^{s j} d_{i j} \lambda^{r i}-\omega^{r i} d_{i j} \lambda^{s j}\right) \Omega_{r s}=\sigma^{r s} \Omega_{r s}
$$

where $\square$ is the standard Dalembertian. Accordingly, we have:

$$
d_{r} \sigma^{r s}=\omega^{i j} d_{r i j} \lambda^{r s}+\omega^{r s} d_{r i j} \lambda^{i j}-\omega^{s j} d_{r i j} \lambda^{r i}-\omega^{r i} d_{r i j} \lambda^{s j}=0
$$

The basic idea used in GR has been to simplify these equations by adding the differential constraints $d_{r} \lambda^{r s}=0$ in order to find only $\square \lambda^{r s}=\sigma^{r s}$, exactly like in the Lorenz condition for EM. Before going ahead, it is important to notice that when $n=2$, the only Lagrange multiplier $\lambda$ is just the Airy function $\phi$ and, using an integration by parts, we have the identity:

$$
\phi\left(d_{11} \Omega_{22}+d_{22} \Omega_{11}-2 d_{12} \Omega_{12}\right)=d_{22} \phi \Omega_{11}-2 d_{12} \phi \Omega_{12}+d_{11} \phi \Omega_{22}+\operatorname{div}()
$$

providing the Airy parametrization of the Cauchy stress equations:

$$
\sigma^{11}=d_{22} \phi, \sigma^{12}=\sigma^{21}=-d_{12} \phi, \sigma^{22}=d_{11} \phi
$$

where the Airy function has, of course, nothing to do with any perturbation of 
the metric.

However, even if it is clear that the constraints are compatible with the Cauchy equations, we do believe that the following result is not known as it does not contain any reference to the usual Einstein tensor $E_{i j}=R_{i j}-\frac{1}{2} \omega_{i j} \operatorname{tr}(R)$ where $\operatorname{tr}(R)=\omega^{r s} R_{r s}$, which is therefore useless because it contains 6 terms instead of 4 terms only, even though the corresponding operator is self-adjoint.

PROPOSITION 4.C.1: The system made by $\square \lambda^{r s}=\sigma^{r s}$ and $d_{r} \lambda^{r s}=0$ is a relative minimum involutive parametrization of the Cauchy equations describing the formal adjoint of the Killing operator, that is Cauchy =ad (Killing) as operators.

Proof: For each given $s=1,2,3,4$ the system under study is exactly the system used for studying the Lorenz condition in Proposition 4.B.1. Accordingly, nothing has to be changed in the proof of this proposition and we get an involutive second order system with $d_{r} \sigma^{r s}=0$ as only CC in place of the conservation of current. Needless to say that this result has nothing to do with any concept of gauge theory as it is sometimes claimed ([8] [47]).

Q.E.D.

\section{Conclusion}

In 1916, F.S. Macaulay used a new localization technique for studying unmixed polynomial ideals. In 2012, we have generalized this procedure in order to study pure differential modules, obtaining therefore a relative parametrization in place of the absolute parametrization already known for torsion-free modules and equivalent to controllability in the study of OD or PD control systems. Such a result is showing that controllability does not depend on the choice of the control variables, despite what engineers still believe. Meanwhile, we have pointed out the existence of minimum parametrizations obtained by adding, in a convenient but generally not intrinsic way, certain compatible differential constraints on the potentials. We have proved that this is exactly the kind of situation met in control theory, in EM with the Lorenz condition and in GR with gravitational waves. However, the systematic use of adjoint operators and differential duality is proving that the physical meaning of the potentials involved has absolutely nothing to do with the one usually adopted in these domains. Therefore, these results bring the need to revisit the mathematical foundations of Electromagnetism and Gravitation, thus of Gauge Theory and General Relativity, in particular Maxwell and Einstein equations, even if they seem apparently well established.

\section{Conflicts of Interest}

The author declares no conflicts of interest regarding the publication of this paper.

\section{References}

[1] Barakat, M. (2010) Purity Filtration and the Fine Structure of Autonomy. Proceed- 
ings of the 19th International Symposium on Mathematical Theory of Networks and Systems, Budapest, 5-9 July 2010, 1657-1661.

[2] Bjork, J.E. (1993) Analytic D-Modules and Applications. Kluwer Academic Publisher, Dordrecht. https://doi.org/10.1007/978-94-017-0717-6

[3] Pommaret, J.-F. (2001) Partial Differential Control Theory. Kluwer Academic Publisher, Dordrecht. https://doi.org/10.1007/978-94-010-0854-9

[4] Pommaret, J.-F. (2013) Multidimensional Systems and Signal Processing, 26, 405-437. https://doi.org/10.1007/s11045-013-0265-0

[5] Pommaret, J.-F. (2005) Algebraic Analysis of Control Systems Defined by Partial Differential Equations. In: Advanced Topics in Control Systems Theory, Lecture Notes in Control and Information Sciences LNCIS 311, Chapter 5, Springer, Berlin, 155-223. https://doi.org/10.1007/11334774_5

[6] Pommaret, J.-F. (2014) Journal of Modern Physics, 5, 157-170. https://doi.org/10.4236/jmp.2014.55026

[7] Pommaret, J.-F. (2018) From Elasticity to Electromagnetism: Beyond the Mirror. http://arxiv.org/abs/1802.02430

[8] Pommaret, J.-F. (2013) Journal of Modern Physics, 4, 223-239. https://doi.org/10.4236/jmp.2013.48A022

[9] Pommaret, J.-F. (2016) Journal of Modern Physics, 7, 699-728. https://doi.org/10.4236/jmp.2016.77068

[10] Pommaret, J.-F. (2017) Journal of Modern Physics, 8, 2122-2158. https://doi.org/10.4236/jmp.2017.813130

[11] Quadrat, A. (2010) Les Cours du CIRM, 1, 281-471. https://doi.org/10.5802/ccirm.11

[12] Quadrat, A. (2013) Acta Applicandae Mathematicae, 127, 27-86. https://doi.org/10.1007/s10440-012-9791-2 http://hal.inria.fr/inria-00632281/fr http://pages.saclay.inria.fr/alban.quadrat/PurityFiltration.html

[13] Pommaret, J.-F. (2018) New Mathematical Methods for Physics. NOVA Science Publisher, New York.

[14] Pommaret, J.-F. (1994) Partial Differential Equations and Group Theory: New Perspectives for Applications. Kluwer Academic Publisher, Dordrecht. https://doi.org/10.1007/978-94-017-2539-2

[15] Pommaret, J.-F. (1995) Comptes rendus de PAcadémie des Sciences, Paris, 320, 1225-1230.

[16] Kalman, E.R., Yo, Y.C. and Narenda, K.S. (1963) Contributions to Differential Equations, 1, 189-213.

[17] Pommaret, J.-F. (2010) Acta Mechanica, 215, 43-55. https://doi.org/10.1007/s00707-010-0292-y

[18] Pommaret, J.-F. (2017) Algebraic Analysis and Mathematical Physics. http://arxiv.org/abs/1706.04105

[19] Pommaret, J.-F. (2019) Journal of Modern Physics, 10, 371-401. https://doi.org/10.4236/jmp.2019.103025

[20] Pommaret, J.-F. and Quadrat, A. (1999) IMA Journal of Mathematical Control and Informations, 16, 275-297. https://doi.org/10.1093/imamci/16.3.275

[21] Macaulay, F.S. (1964) The Algebraic Theory of Modular Systems, Cambridge Tracts, Vol. 19, Cambridge University Press, London, 1916. Stechert-Hafner Service 
Agency, New York.

[22] Bourbaki, N. (1985) Algèbre Commutative, Chap. I-IV. Masson, Paris.

[23] Bourbaki, N. (1980) Algèbre Homologique, Chap. X. Masson, Paris.

[24] Hu, S.-T. (1968) Introduction to Homological Algebra, Holden-Day.

[25] Kashiwara, M. (1995) Algebraic Study of Systems of Partial Differential Equations, Mémoires de la Société Mathématique de France 63. Transl. from Japanese of His 1970 Master's Thesis.

[26] Kunz, E. (1985) Introduction to Commutative Algebra and Algebraic Geometry, Birkhäuser. https://doi.org/10.1007/978-1-4612-5290-0

[27] Maisonobe, P. and Sabbah, C. (1993) D-Modules Cohérents et Holonomes, Travaux en Cours. Hermann, Paris, 45.

[28] Northcott, D.G. (1966) An Introduction to Homological Algebra. Cambridge University Press, Cambridge.

[29] Northcott, D.G. (1968) Lessons on Rings, Modules and Multiplicities. Cambridge University Press, Cambridge. https://doi.org/10.1017/CBO9780511565922

[30] Rotman, J.J. (1979) An Introduction to Homological Algebra, Pure and Applied Mathematics. Academic Press, Cambridge.

[31] Schneiders, J.-P. (1994) Bulletin de la Société Royale des Sciences de Liège, 63, 223-295.

[32] Pommaret, J.-F. (1978) Systems of Partial Differential Equations and Lie Pseudogroups. Gordon and Breach, New York (Russian Translation: MIR, Moscow, 1983).

[33] Pommaret, J.-F. (1983) Differential Galois Theory. Gordon and Breach, New York.

[34] Pommaret, J.-F. (2016) Deformation Theory of Algebraic and Geometric Structures. Lambert Academic Publisher, (LAP), Saarbrucken. http://arxiv.org/abs/1207.1964

[35] Janet, M. (1920) Journal d Analyse Mathematique, 8, 65-151.

[36] Seiler, W.M. (2009) Involution: The Formal Theory of Differential Equations and Its Applications to Computer Algebra. Springer, Berlin, $660 \mathrm{p}$.

https://doi.org/10.1007/978-3-642-01287-7_2

[37] Pommaret, J.-F. and Quadrat, A. (1999) Systems \& Control Letters, 37, 247-260. https://doi.org/10.1016/S0167-6911(99)00030-4

[38] Quadrat, A. (1999) Analyse Algébrique des Systèmes de Contrôle Linéaires Multidimensionnels. Thèse de Docteur de l'Ecole Nationale des Ponts et Chaussées. http://www-sop.inria.fr/cafe/Alban.Quadrat/index.html

[39] Pommaret, J.-F. (2011) Journal of Symbolic Computation, 46, 1049-1069. https://doi.org/10.1016/j.jsc.2011.05.007

[40] Pommaret, J.-F. (1988) Lie Pseudogroups and Mechanics. Gordon and Breach, New York.

[41] Pommaret, J.-F. (2012) Spencer Operator and Applications: From Continuum Mechanics to Mathematical Physics. In: Gan, Y., Ed., Continuum Mechanics-Progress in Fundamentals and Engineering Applications, InTech, London, 1-32. https://doi.org/10.5772/35607 http://www.intechopen.com/books/continuum-mechanics-progress-in-fundamenta ls-and-engineerin-applications/spencer-operator-and-applications-from-continuu m-mechanics-to-mathematical-physics

[42] Spencer, D.C. (1965) Bulletin of the American Mathematical Society, 75, 1-114.

[43] Zerz, E. (2000) Topics in Multidimensional Linear Systems Theory. Lecture Notes 
in Control and Information Sciences, LNCIS 256, Springer, Berlin.

[44] Cahen, M. and Gutt, S. (1981) Bulletin de la Soc. Mathématique de Belgique, 33, 91-97.

[45] Pommaret, J.-F. (2016) From Thermodynamics to Gauge Theory: The Virial Theorem Revisited. In: Bailey, L., Ed., Gauge Theories and Differential Geometry, NOVA Publishers, New York, Chapter I, 1-44.

[46] Cosserat, E. and Cosserat, F. (1909) Théorie des Corps Déformables. Hermann, Paris.

[47] Foster, J. and Nightingale, J.D. (1979) A Short Course in General Relativity. Longman, Harlow. 


\section{Main Notations}

$K$ differential field containing $\mathbb{Q}$ with $n$ commuting derivations $\partial_{1}, \cdots, \partial_{n}$. $d_{1}, \cdots, d_{n}$ formal derivatives acting on the $m$ differential indeterminates $y^{1}, \cdots, y^{m}$. $D=K\left[d_{1}, \cdots, d_{n}\right]=K[d]$ ring of differential operators $P, Q$ with coefficients in $K$.

$L, M, N$ filtered differential modules over $D$.

$\mu=\left(\mu_{1}, \cdots, \mu_{n}\right)$ multi-index with length $|\mu|=\mu_{1}+\cdots+\mu_{n}$ and $\mu+1_{i}=\left(\mu_{1}, \cdots, \mu_{i}+1, \cdots, \mu_{n}\right)$.

$X$ manifold with tangent, cotangent, symmetric and exterior bundles

$T, T^{*}, S_{q} T^{*}, \wedge^{r} T^{*}$.

$y_{q}=\left(y^{k}, y_{i}^{k}, y_{i j}^{k}, \cdots\right)=\left(y_{\mu}^{k}\right), 0 \leq|\mu| \leq q$ jet coordinates up to order $q$.

$\operatorname{ad}(\mathcal{D})$ formal adjoint defined by $\langle\lambda, \mathcal{D} \xi\rangle=\langle\operatorname{ad}(\mathcal{D}) \lambda, \xi\rangle+\operatorname{div}()$ for any test row vector $\lambda$.

$E, F$ vector bundles over $X$ or free modules over $D$.

$V$ characteristic algebraic variety with dimension $d$ and codimension $c d=n-d$ over $K$.

$0=t_{n}(M) \subseteq \cdots \subseteq t_{0}(M) \subseteq M$ purity filtration with $t_{r}(M)=\{m \in M \mid c d(D m)>r\}$.

$t_{0}(M)=t(M)=\{m \in M \mid \exists 0 \neq P \in D, P m=0\}$ the torsion submodule of $M$. $R=\operatorname{hom}_{K}(M, K)$ differential module over $D$ associated with $M$, also called inverse system.

$d: R \rightarrow T^{*} \otimes_{K} R=f \rightarrow d x^{i} \otimes d_{i} f$ Spencer operator with $\left(d_{i} f\right)_{\mu}^{k}=\partial_{i} f_{\mu}^{k}-f_{\mu+1_{i}}^{k}$. $T^{*} \otimes_{X} T^{*} \simeq S_{2} T^{*} \oplus \wedge^{2} T^{*}$ with $R=\left(R_{i j}\right) \in S_{2} T^{*} \quad$ Ricci tensor and $F=\left(F_{i j}\right) \in \wedge^{2} T^{*}$ EM field. 